\title{
Chaotic Characteristics and Application of Cooperative Game and Evolutionary Game
}

\author{
Yujing Yang, Junhai Ma, and Hongliang Tu \\ College of Management Economic, Tianjin University, Tianjin, China \\ Correspondence should be addressed to Junhai Ma; mjhtju@aliyun.com
}

Received 2 October 2013; Accepted 26 December 2013; Published 19 March 2014

Academic Editor: Zbigniew Leśniak

Copyright ( 2014 Yujing Yang et al. This is an open access article distributed under the Creative Commons Attribution License, which permits unrestricted use, distribution, and reproduction in any medium, provided the original work is properly cited.

\begin{abstract}
According to a dynamical multiteam Cournot game in exploitation of a renewable resource, a new dynamic Cournot duopoly game model with team players in exploitation of a renewable resource is built up in this paper. Based on the theory of bifurcations of dynamical systems, the stability of the system is studied and the local stable region of Nash equilibrium point is obtained. The effect of the output adjustment speed parameters and the weight parameter of the system on the dynamic characteristics of the system are researched. The complexity of the system is described via the bifurcation diagrams, the Lyapunov exponents, the phase portrait, the time history diagram, and the fractal dimension. Furthermore, the chaos control of the system is realized by the parameter adjustment method. At last, an evolutionary game as a special dynamic system is constructed and analyzed which is more useful and helpful in application. The derived results have very important theoretical and practical values for the renewable resource market and companies.
\end{abstract}

\section{Introduction}

Chaos has become a hot topic in the competition of oligarchs. Research on the complexity of the oligopoly game model has been paid attention to by researchers and scholars recently. Reference [1] studied different strategies which are the Cournot model, the Stackelberg case, and the dynamic system of a duopoly game and investigated stable equilibrium point, cycles, bifurcation, and chaos of the systems. Reference [2] obtained the explicit stability zones for Cournot game with 3 and 4 competitors, and [3] analyzed the stability, bifurcation, chaos, and chaos control of a Kopel model. Reference [4] studied the chaotic dynamics in nonlinear duopoly game with bounded and naive players. Reference [5] constructed a stochastic optimal control model of pollution abatement and studied the control of the model. Reference [6] studied the dynamical behaviors of a duopoly game with delayed bounded rationality and obtained some practical and theoretical significance in the practice. Reference [7] studied the complex dynamics in nonlinear triopoly market with different expectations and obtained some useful conclusions. Reference $[8,9]$ made the analysis on the complexity of a Cournot-Bertrand duopoly game model with limited information and introduced a 4D Hyperchaotic System, making the numerical simulation and achieving a second control. Reference [10] introduced a social behavior selection model for evolutionary dynamics of behaviors in social networks which exhibits a rich set of emergent behaviors of evolution and studied the chaotic dynamics of the social behavior selection networks in crowd simulation. Reference [11] studied the dynamics and adaptive control of a duopoly advertising model based on heterogeneous expectations and gave the scope of the convergent condition and control intensity. And the result demonstrated that the adaptive controlled method could lead the chaos track to low cycle track. In particular, cooperation and multiteam games had become popular in the fields of chaos in recent years. Reference [12] made analysis on whether the migration cost influences cooperation among success-driven individuals. Reference [13] studied cooperation in an evolutionary prisoner's dilemma game with probabilistic strategies. Reference [14] made analysis on dynamical multiteam and signaling games. References $[15,16]$ formulated the standard and multiteam Bertrand game which is based on Puu's incomplete information and 
studied quantum team games, respectively. Reference [17] proposed two different versions of the multiteam model: one is that the firms in each team can help each other, and the other is that they competed with another team. Finally, the conditions of their local asymptotic stability were studied. Reference [18] studied an incomplete information dynamical system and a modification of the system was suggested and applied to the standard Cournot game and formulated and studied the multiteam dynamic Cournot game. Reference [19] mainly established a dynamical multiteam Cournot game in exploitation of a renewable resource and analyzed the asymptotic stability of the equilibrium solution of the game. It is worthy to further analyze and extend it in this area. Reference [20] analyzed the purely competitive evolutionary dynamics for games and proposed that perhaps the system will enter a chaotic state for some parameters.

Reference [19-24] have analyzed the dynamic characters in the economic fields, and drew some valuable conclusions.

The structures of this paper are as follows. In Section 2, the dynamic Cournot duopoly game model is established. In Section 3, the existence and the local stability of the Nash equilibrium point are studied, and the stable region of the Nash equilibrium point is derived. In Section 4, dynamical characteristics of the system are analyzed. The effect of the output adjustment speed parameters and the weight parameter on the dynamics behaviors of the system is discussed. Numerical simulation method is used to show complex dynamics of the system by means of the bifurcation diagram, the Lyapunov exponents, the phase portrait, the time history diagram, and the fractal dimension. In Section 5, bifurcation and chaos control of the model are used by the parameter adjustment method. In Section 6, a model of evolutionary game is analyzed by numerical simulation. We obtain the conclusion that evolutionary game is a special dynamic by analyzing the change of Lyapunov exponents. Finally, some results are summarized.

\section{The Model}

Suppose that there are two mainly oligopoly companies $X_{1}$, $X_{2}$ in exploitation of a renewable resource. The company $X_{i}(i=1,2)$ makes the optimal output decision, and suppose that the $t$-output is $q_{i}(t)(i=1,2)$. At each period $t$, the price $p$ is determined by the total output $Q_{T}(t)=q_{1}(t)+q_{2}(t)$.

According to [19], we also propose the renewable resource market with the linear inverse demand function:

$$
p(t)=a-b Q_{T}(t),
$$

and the cost function of the company $X_{i}(i=1,2)$ is as follows:

$$
C_{i}(t)=c_{i}+\frac{d_{i} q_{i}^{2}(t)}{Q_{T}(t)}, \quad(i=1,2),
$$

where $c_{i}(i=1,2)$ is the fixed cost and $d_{i}(i=1,2,3)$ is positive parameter.
We can get the profit of the company $X_{i}(i=1,2)$ as follows:

$$
\pi_{i}(t)=\left[a-b Q_{T}(t)\right] q_{i}(t)-c_{i}-\frac{d_{i} q_{i}^{2}(t)}{Q_{T}(t)}, \quad(i=1,2) .
$$

Here, it is assumed that the renewable resource firms $X_{1}$, $X_{2}$ establish a team, and the profit of the team is

$$
\begin{aligned}
\pi^{\text {team }}(t)= & \theta\left[\left(a-b Q_{T}(t)\right) q_{1}(t)-c_{1}-\frac{d_{1} q_{1}^{2}(t)}{Q_{T}(t)}\right] \\
& +(1-\theta)\left[\left(a-b Q_{T}(t)\right) q_{2}(t)-c_{2}-\frac{d_{2} q_{2}^{2}(t)}{Q_{T}(t)}\right],
\end{aligned}
$$

where the $\theta$ is the weight parameter of the profit of the renewable resource company $X_{1}$ in the team. We propose that the company $X_{i}(i=1,2)$ takes bounded rational strategy under the maximum of team profit. Since the game between the companies is a continuous and long-term repeated dynamical process, the dynamical adjustment of the player in the duopoly game can be expressed as follows:

$$
q_{i}(t+1)=q_{i}(t)+\alpha_{i} q_{i}(t) \frac{\partial \pi^{\text {team }}(t)}{\partial q_{i}(t)}, \quad(i=1,2),
$$

where $\alpha_{i}(i=1,2)$ is the output adjustment speed parameter.

Combining (4) and (5), a new dynamic Cournot duopoly game with team players in exploitation of a renewable resource is obtained. This map has the following form:

$$
\begin{aligned}
& q_{1}(t+1) \\
& =q_{1}(t)+\alpha_{1} q_{1}(t) \\
& \times\left[\theta\left(a-b Q_{T}(t)-b q_{1}-\frac{2 d_{1} q_{1}(t)}{Q_{T}(t)}+\frac{d_{1} q_{1}^{2}(t)}{Q_{T}^{2}(t)}\right)\right. \\
& \left.+(1-\theta)\left(-b q_{2}(t)+\frac{d_{2} q_{2}^{2}(t)}{Q_{T}^{2}(t)}\right)\right], \\
& q_{2}(t+1) \\
& =q_{2}(t)+\alpha_{2} q_{2}(t) \\
& \times\left[\theta\left(-b q_{1}(t)+\frac{d_{1} q_{1}^{2}(t)}{Q_{T}^{2}(t)}\right)\right. \\
& \left.+(1-\theta)\left(a-b Q_{T}(t)-b q_{2}-\frac{2 d_{2} q_{2}(t)}{Q_{T}(t)}+\frac{d_{2} q_{2}^{2}(t)}{Q_{T}^{2}(t)}\right)\right] .
\end{aligned}
$$

\section{The Stability of the System}

In system (6), $\alpha_{i}(i=1,2,3)$ is taken as bifurcation parameter, and the other parameters are as follows: $a=6.29, b=0.92$, $d_{1}=0.265, d_{2}=0.374, d_{3}=0.436$, and $\theta=0.5$. 
Letting the marginal profits be equal to 0 , we can get the Nash equilibrium point. The fixed points of system (6) satisfy the following equations:

$$
\begin{aligned}
& q_{1}\left[\theta\left(a-b Q_{T}-b q_{1}-\frac{2 d_{1} q_{1}}{Q_{T}}+\frac{d_{1} q_{1}^{2}}{Q_{T}^{2}}\right)\right. \\
& \left.+(1-\theta)\left(-b q_{2}+\frac{d_{2} q_{2}^{2}}{Q_{T}^{2}}\right)\right]=0, \\
& q_{2}\left[\theta\left(-b q_{1}+\frac{d_{1} q_{1}^{2}}{Q_{T}^{2}}\right)+(1-\theta)\right. \\
& \left.\quad \times\left(a-b Q_{T}-b q_{2}-\frac{2 d_{2} q_{2}}{Q_{T}}+\frac{d_{2} q_{2}^{2}}{Q_{T}^{2}}\right)\right]=0 .
\end{aligned}
$$

The equations [21] are solved and three meaningful fixed points $p_{1}(0.8651,0.7369), p_{2}(1.546875,0)$, and $p_{3}(0,1.5260)$ are obtained. Here, we only consider the stability of the Nash equilibrium point $p_{1}\left(q_{1}^{*}=0.8651, q_{2}^{*}=0.7369\right)$ and denote the total output at the Nash equilibrium point as $Q_{T}^{*}=q_{1}^{*}+q_{2}^{*}$.

We can calculate the Jacobian matrix of system (6) at the Nash equilibrium point $p_{1}$ :

$$
J=\left(\begin{array}{cc}
1+j_{11} & j_{12} \\
j_{21} & 1+j_{22}
\end{array}\right)
$$

where

$$
\begin{aligned}
& j_{11}=\alpha_{1} q_{1}^{*}[ \theta\left(-2 b-\frac{2 d_{1}}{Q_{T}^{*}}+\frac{4 d_{1} q_{1}^{*}}{Q_{T}^{* 2}}-\frac{2 d_{1} q_{1}^{* 2}}{Q_{T}^{* 3}}\right) \\
&\left.+(1-\theta) \frac{2 d_{2} q_{2}^{* 2}}{Q_{T}^{* 3}}\right], \\
& j_{12}=\alpha_{1} q_{1}^{*}\left[\theta\left(-b+\frac{2 d_{1} q_{1}^{*}}{Q_{T}^{* 2}}-\frac{2 d_{1} q_{1}^{* 2}}{Q_{T}^{* 3}}\right)\right. \\
&\left.+(1-\theta)\left(-b+\frac{2 d_{2} q_{2}^{*}}{Q_{T}^{* 2}}-\frac{2 d_{2} q_{2}^{* 2}}{Q_{T}^{* 3}}\right)\right], \\
& j_{21}=\alpha_{2} q_{2}^{*}\left[\theta\left(-b+\frac{2 d_{1} q_{1}^{*}}{Q_{T}^{* 2}}-\frac{2 d_{1} q_{1}^{* 2}}{Q_{T}^{* 3}}\right)\right. \\
&\left.+(1-\theta)\left(-b+\frac{2 d_{2} q_{2}^{*}}{Q_{T}^{* 2}}-\frac{2 d_{2} q_{2}^{* 2}}{Q_{T}^{* 3}}\right)\right], \\
& j_{22} \alpha_{2} q_{2}^{*}\left[-\theta \frac{2 d_{1} q_{1}^{* 2}}{Q_{T}^{* 3}}+(1-\theta)\right. \\
&\left.\times\left(-2 b-\frac{2 d_{2}}{Q_{T}^{*}}+\frac{4 d_{2} q_{2}^{*}}{Q_{T}^{* 2}}-\frac{2 d_{2} q_{2}^{* 2}}{Q_{T}^{* 3}}\right)\right] .
\end{aligned}
$$

Furthermore, we can get the characteristic polynomial of system (6) at $p_{1}$ :

$$
f(\lambda)=\lambda^{2}+A_{1} \lambda+A_{0},
$$

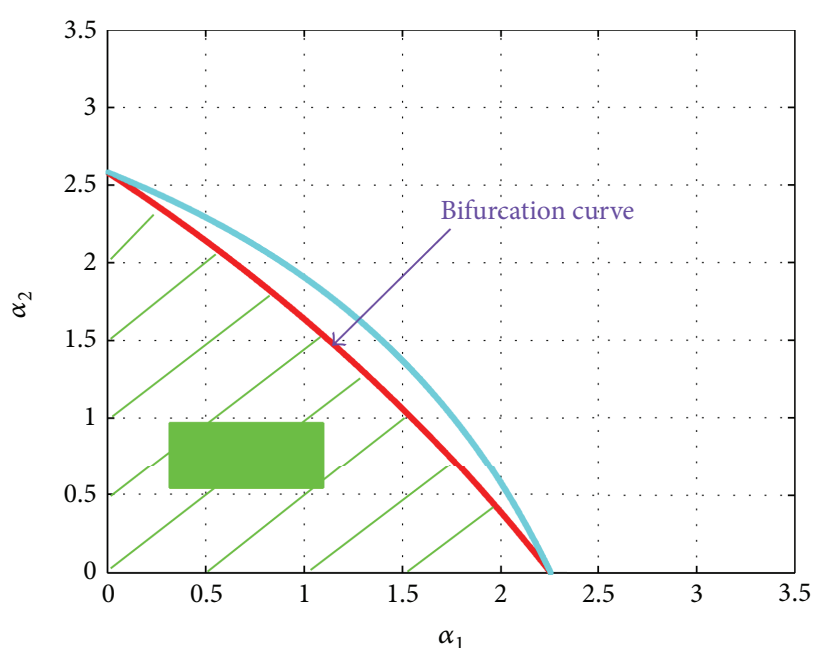

FIGURE 1: The stable region of Nash equilibrium point about $\alpha_{1}$ and $\alpha_{2}$.

where

$$
\begin{gathered}
A_{1}=-\left(2+j_{11}+j_{22}\right), \\
A_{0}=\left(1+j_{11}\right)\left(1+j_{22}\right)-j_{12} j_{21} .
\end{gathered}
$$

According to the Jury test [21], the necessary and sufficient condition of the local stability of Nash equilibrium should satisfy the following three conditions:

(i) $1+A_{1}+A_{0}>0$,

(ii) $1-A_{1}+A_{0}>0$,

(iii) $\left|A_{0}\right|<1$.

The local stable region of Nash equilibrium point can be obtained by solving the above equations. The stable region of Nash equilibrium point about the output adjustment speed parameter $\alpha_{i}(i=1,2)$ is shown in Figure 1 . The Nash equilbrium is stable for the values of $\alpha_{1}$ and $\alpha_{2}$ inside the stable region. The meaning of the stable region is that both the two renewable resource companies will eventually maintain at Nash equilibrium output after finite games whatever initial output is chosen in the local stable region. It is valuable to study that the renewable resource companies increase the output adjustment speed in order to get more profit. Though output adjustment speed parameters are unrelated to the Nash equilibrium point, the system will become unstable and even fall into chaos if one renewable resource company that adjusts output speed is too fast and pushes the output adjustment speed parameter $\alpha_{i}(i=1,2)$ out of the stable region. Numerical simulation method is used to analyze the dynamic characteristics of system (6) with the change of $\alpha_{i}(i=1,2)$. Numerical results such as the bifurcation diagrams, the strange attractors, the Lyapunov exponents, the sensitive dependence on initial conditions, and the fractal structure will be considered in the following section. 


\section{Dynamic Characteristics of the System}

In this game, the renewable resource companies make the optimal output decision to get the maximum profit and adjust their output based on the marginal profit of last period. In the oligopoly market, the players have the driving force to increase their output in the hope of achieving more profits. The renewable resource firms can adjust their output speed to increase their output. So, the output adjustment speed parameter $\alpha_{i}(i=1,2)$ affects the game results very much. The output adjustment speed parameter $\alpha_{i}(i=1,2)$ affects the dynamic behaviors of system (6), which will be analyzed in the following section.

4.1. The Effect of Output Adjustment Speed on the System. The stability of Nash equilibrium point will change if company $X_{1}$ accelerates the output adjustment speed and pushes $\alpha_{1}$ out of the stable region. When $\alpha_{2}=0.146$, with the output adjustment speed $\alpha_{1}$ increasing, the output evolution of the duopoly starts with equilibrium state, through period doubling bifurcation, and ends with chaotic state. From the bifurcation diagram in Figure 2 and the corresponding Lyapunov exponents diagram in Figure 3, we can see that $\alpha_{1} \epsilon$ $(0,1.1654]$ is the domain of system (6) in equilibrium state as shown in Figure $4, \alpha_{1} \in(1.1654,1.7200]$ is the domain of 2 period orbits of system (6), and $\alpha_{1} \in(1.7200,1.8556]$ is the domain of 4 period orbits of system (6), $\alpha_{1} \in(1.8556,1.8925]$ is the domain of 8 period orbits of system (6), for $\alpha_{1} \epsilon$ $(1.8925,2.2622]$, system $(6)$ is in a chaotic state. Calculation of the Lyapunov exponent is used to analyze the quantitative characteristics of the dynamic system. The system is in chaotic state if the largest Lyapunov exponent is positive. In addition, the larger the positive Lyapunov exponent is, the more obvious the chaotic state of the system is. Figure 5 shows the representative chaotic attractor at initial point $\left(q_{1_{0}}=\right.$ $\left.0.56, q_{2_{0}}=0.96\right)$ and $\alpha_{2}=1.46$, and $\alpha_{1}$ equals 2.13 .

Furthermore, the bifurcation diagram in Figure 6 and the corresponding Lyapunov exponents diagram in Figure 7 show a one-parameter bifurcation diagram with respect to $\alpha_{2}$ when $\alpha_{1}$ is 1.32 . We can see that $\alpha_{2} \in(0,1.2751]$ is the domain of system (6) in equilibrium state. $\alpha_{2} \epsilon$ $(1.2751,2.0362]$ is the domain of 2 period orbits of system (6). $\alpha_{2} \in(2.0362,2.2617]$ is the domain of 4 period orbits of system (6). $\alpha_{2} \in(2.2617,2.3181]$ is the domain of 8 period orbits of system (6). For $\alpha_{2} \in(2.3181,2.51538]$, system (6) is in a chaotic state. Figure 8 shows the representative chaotic attractor at initial point $\left(q_{1_{0}}=0.76, q_{2_{0}}=0.92\right)$ and $\alpha_{1}=1.32$, and $\alpha_{2}$ equals 2.471 .

From the analysis above, we can find that the stability of system (6) will be changed and even the complex dynamic behaviors occur with the increase of the output adjustment speed $\alpha_{i}(i=1,2,3)$.

4.2. The Weight Parameter $\theta$ Effects on the System. In this section, we study the weight parameter $\theta$ which affects the dynamic behaviors of system (6).

Likewise, the bifurcation diagram in Figure 9 and the corresponding Lyapunov exponents diagram in Figure 10 shows

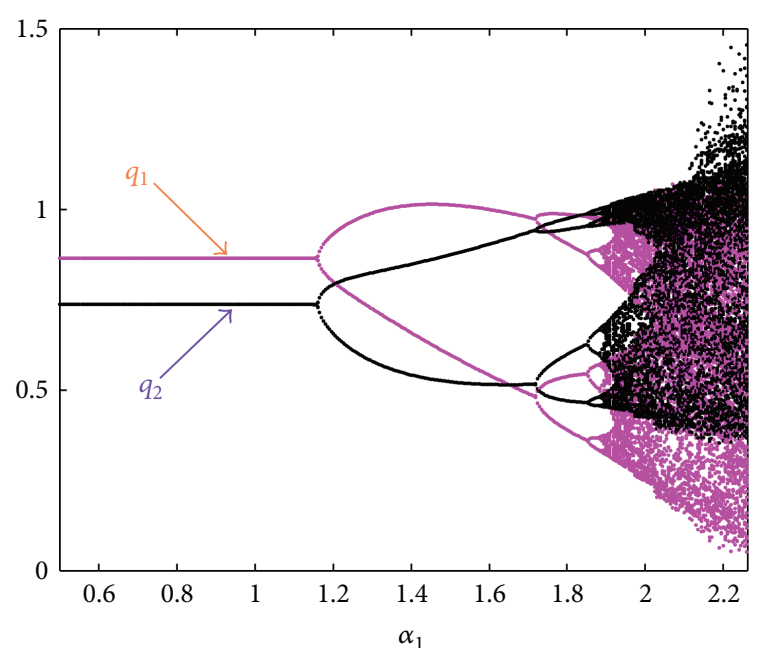

FIGURE 2: Bifurcation diagram of system (6) with $\alpha_{1} \in(0.5,2.2622]$ and $\alpha_{2}=1.46$.

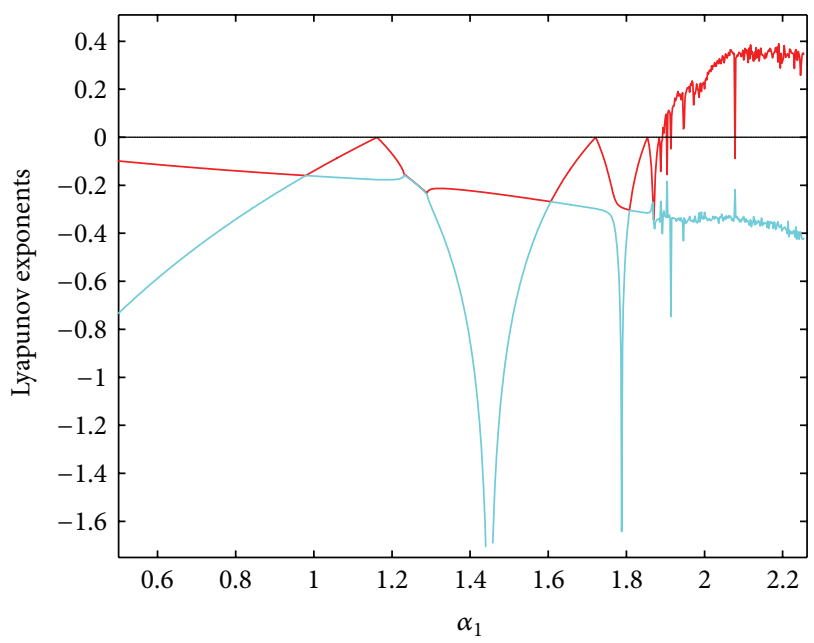

FIGURE 3: The corresponding Lyapunov exponents diagram with $\alpha_{1} \in(0.5,2.2622]$ and $\alpha_{2}=1.46$.

a one-parameter bifurcation diagram with respect to $\theta$ when $\alpha_{1}=1.02$ and $\alpha_{2}=1.46 . \theta \in(0.40731,0.575236]$ is the domain which has economic meaning. One can see that system (6) is stable at different Nash equilibrium points when $\theta \in(0.47645,0.575236]$; that is to say, the duopoly renewable resource companies are in different equilibrium states. $\theta \in$ $(0.40731,0.47645]$ is the domain of 2 period orbits of system (6) as shown in Figure 11. In both sides of the stable domain, system (6) undergoes period doubling bifurcations to chaos with the weight parameter $\theta$ decreasing and increasing, which only has dynamic meaning, and they do not have economic meaning. For $\theta \in(0,0.392489]$, the output $q_{1}=0$, but the output $q_{2}$ is in chaotic state. For $\theta \in(0.392489,0.397427]$, the output $q_{1}=0$, but the output $q_{2}$ has 8 -cycle output orbits as shown in Figure 13. For $\theta \in(0.397427,0.407306]$, the output $q_{1}=0$, but the output $q_{2}$ has 4 -cycle output orbits as shown in Figure 12. For $\theta \in(0.575235,0.659201]$, the output $q_{2}=0$, but the output $q_{1}$ has different equilibrium states. 


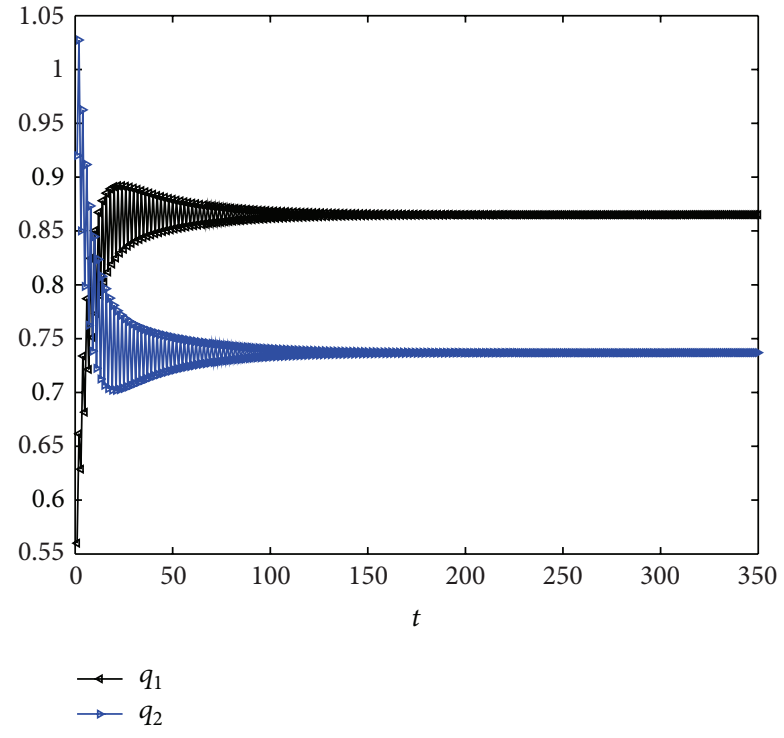

FIGURE 4: For $\alpha_{1}=1.13$ and $\alpha_{2}=1.46$, the wave plots of system (6) with the change of time.

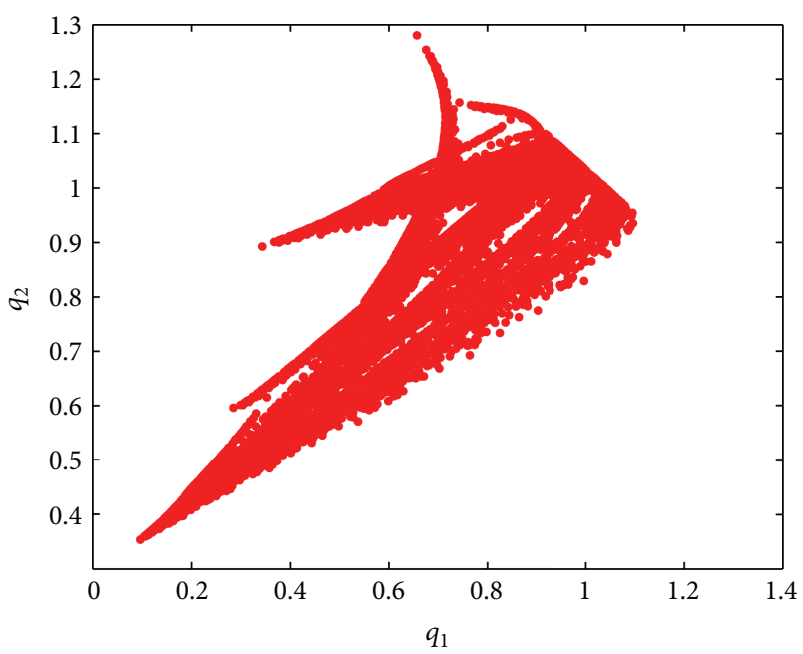

FIGURE 5: Chaos attractor of system (6) for $\alpha_{1}=2.13$ and $\alpha_{2}=1.46$.

For $\theta \in(0.659201,0.782679]$, the output $q_{2}=0$, but the output $q_{1}$ has 2-cycle output orbits as shown in Figure 14 . For $\theta \in(0.782679,0.841948]$, the output $q_{2}=0$, but the output $q_{1}$ have 4-cycle output orbits as shown in Figure 15. For $\theta \in(0.841948,0.846887]$, the output $q_{2}=0$, but the output $q_{1}$ has 8-cycle output orbits as shown in Figure 16. For $\theta \in(0.846887,1]$, the output $q_{2}=0$, but the output $q_{1}$ is in chaotic state as shown in Figure 17.

From the analysis above, we can see that the weight parameter $\theta$ not only affects the stability of system (6), but also changes the Nash equilibrium point. In both sides of the stable domain, system (6) undergoes period bifurcations to chaos with the weight parameter $\theta$ decreasing or increasing, which has no economic meaning.

4.3. Sensitive Dependence on Initial Conditions. One of the most important characteristics of the chaos is extremely

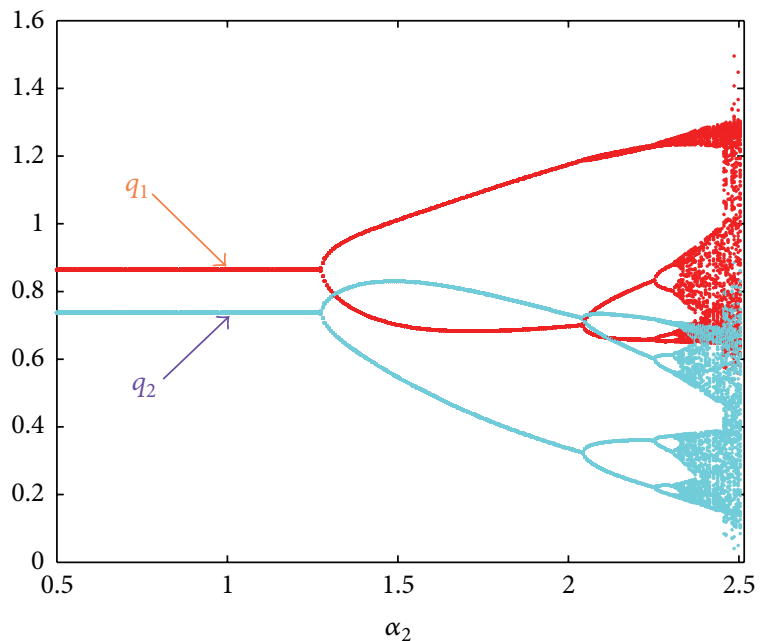

Figure 6: Bifurcation diagram of system (6) with $\alpha_{2} \in(0.5,2.51538]$ and $\alpha_{1}=1.32$.

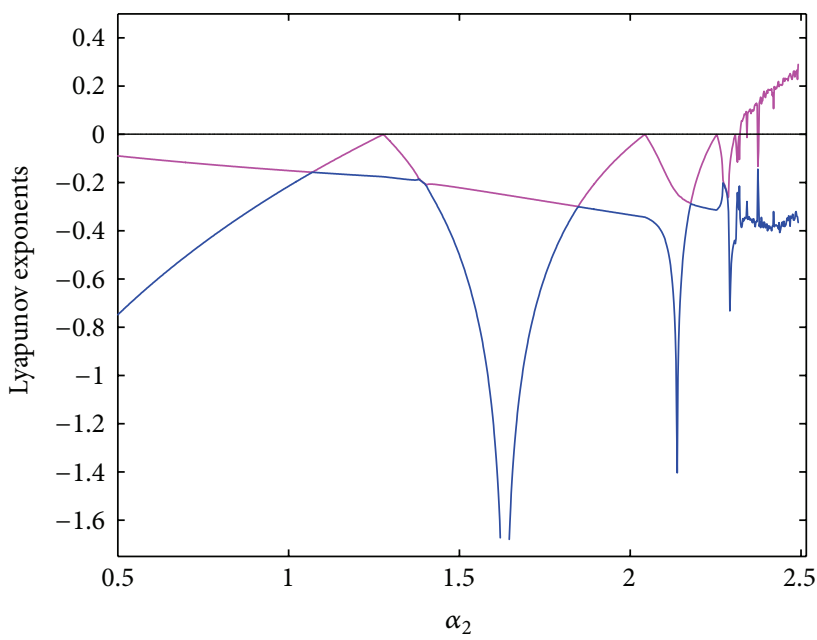

FIGURE 7: The corresponding Lyapunov exponents diagram with $\alpha_{2} \in(0.5,2.51538]$ and $\alpha_{1}=1.32$.

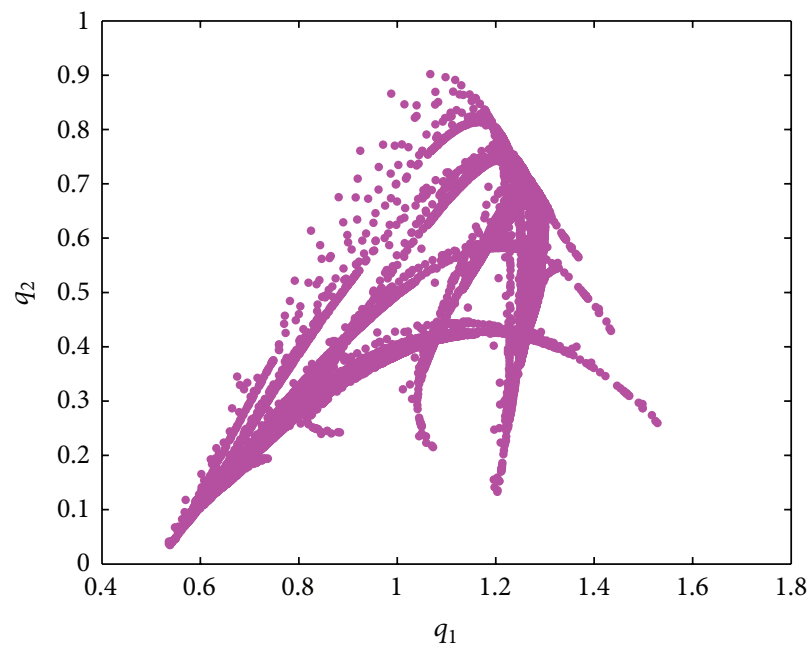

FIGURE 8: Chaos attractors of system (6) for $\alpha_{1}=1.32$ and $\alpha_{2}=$ 2.471 . 


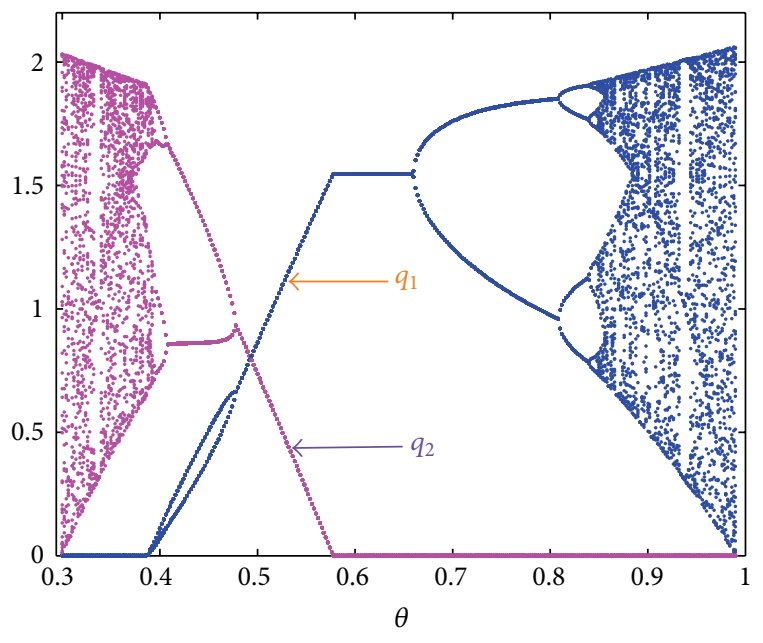

FIGURE 9: Bifurcation diagram of system (6) with $\theta \in(0.2937,1]$ and $\alpha_{1}=1.02$ and $\alpha_{2}=1.46$.

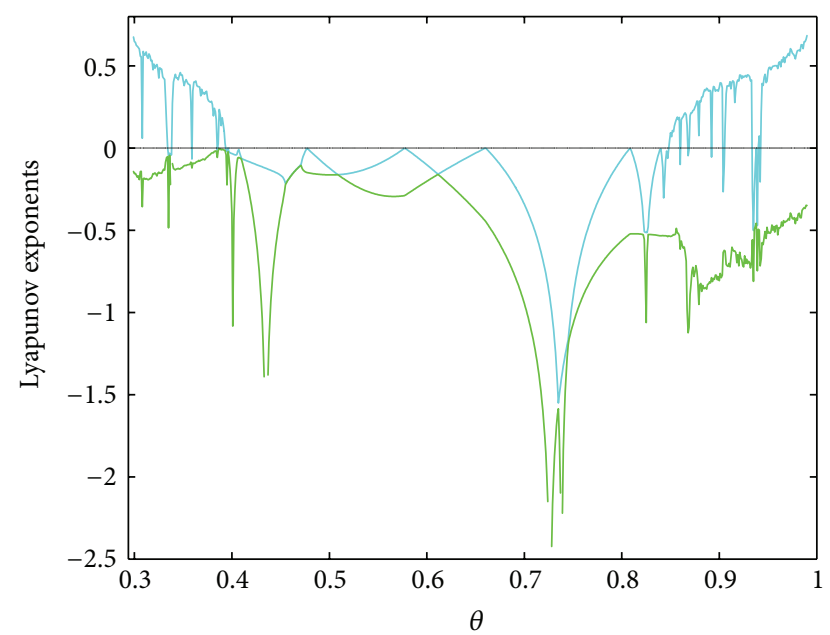

FIGURE 10: The corresponding Lyapunov exponents diagram with $\theta \in(0.2937,1]$ and $\alpha_{1}=1.02$ and $\alpha_{2}=1.46$.

sensitive dependence on initial conditions. Figures 18 and 19 and Figures 20 and 21 show the output history diagram and difference with the change of time when system (6) has different initial conditions. We can see that there is almost no distinction between them in the beginning, but the difference becomes more and more between them with the number of games increasing. It implies that a slight difference between initial values can lead to a great effect on the game results. For $\alpha_{1}=2.05, \alpha_{2}=1.46$ and $\theta=0.5$ and $\alpha_{1}=1.32$, $\alpha_{2}=2.471$ and $\theta=0.5$, it further affirms that system (6) is in a chaotic state. When the system is in a chaotic state, the market will be destroyed and it is difficult for the renewable resource companies to make long-term plan. So, every action from the renewable resource firms can result in a great loss.

4.4. Fractal Dimension. Fractal dimension can be used as another criterion to judge whether the system is in a chaotic state or not. There are many specific definitions of fractal

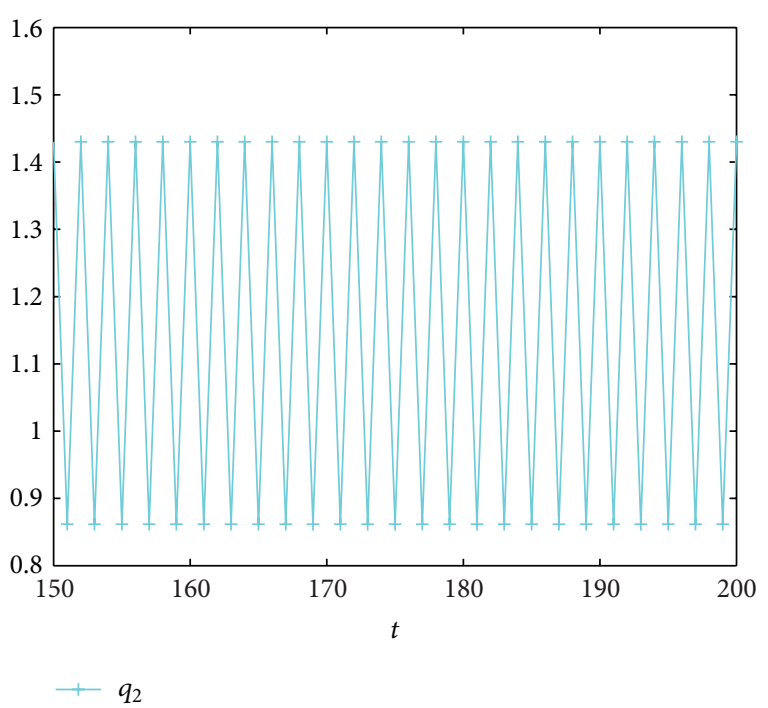

FIGURE 11: The wave plots of the output $q_{2}$ with the change of time when $\alpha_{1}=1.02, \alpha_{2}=1.46$ and $\theta=0.436$.

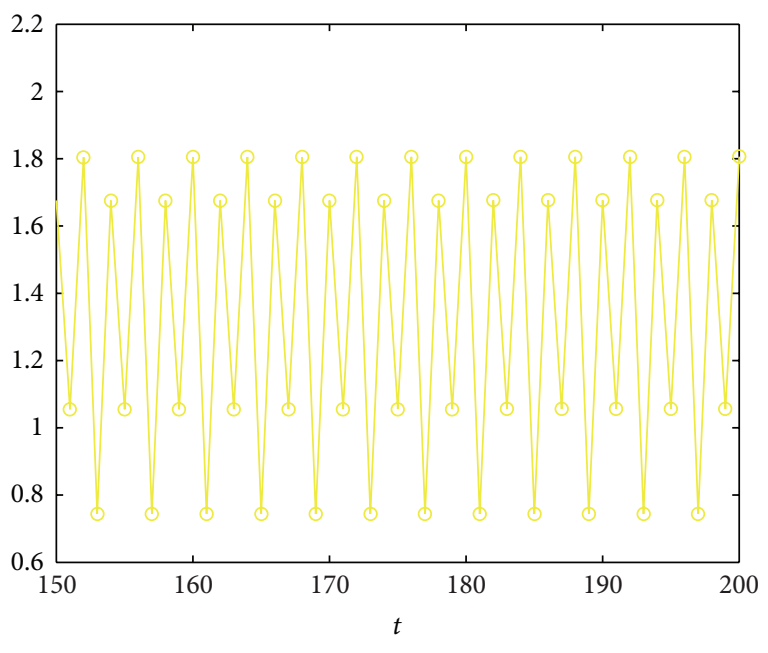

$q_{2}$

FIGURE 12: The wave plots of the output $q_{2}$ with the change of time when $\alpha_{1}=1.02, \alpha_{2}=1.46$ and $\theta=0.397$.

dimension, but none of them can be taken as the universal one. According to [22], the following definition of fractal dimension is adopted in this paper:

$$
d=j-\frac{\sum_{1}^{j} \lambda_{i}}{\lambda_{j+1}}
$$

where $\lambda_{1}>\lambda_{2}>, \ldots, \lambda_{n}$ are the Lyapunov exponents and $j$ is the largest positive integer for which satisfies $\sum_{1}^{j} \lambda_{i}>0$ and $\sum_{1}^{j+1} \lambda_{i}<0$. If $\lambda_{i} \geq 0, i=1,2, \ldots, n$, then $d=n$. If $\lambda_{i}<0$, $i=1,2, \ldots, n$, then $d=0$.

The Lyapunov exponents of system (6) are $\lambda_{1}=0.309959$ and $\lambda_{2}=-0.332619$ for $\alpha_{1}=2.05$ and $\alpha_{2}=1.46$. System (6) is in a chaotic state because the largest Lyapunov exponent $\lambda_{1}$ is positive. Fractal dimension demonstrates that 


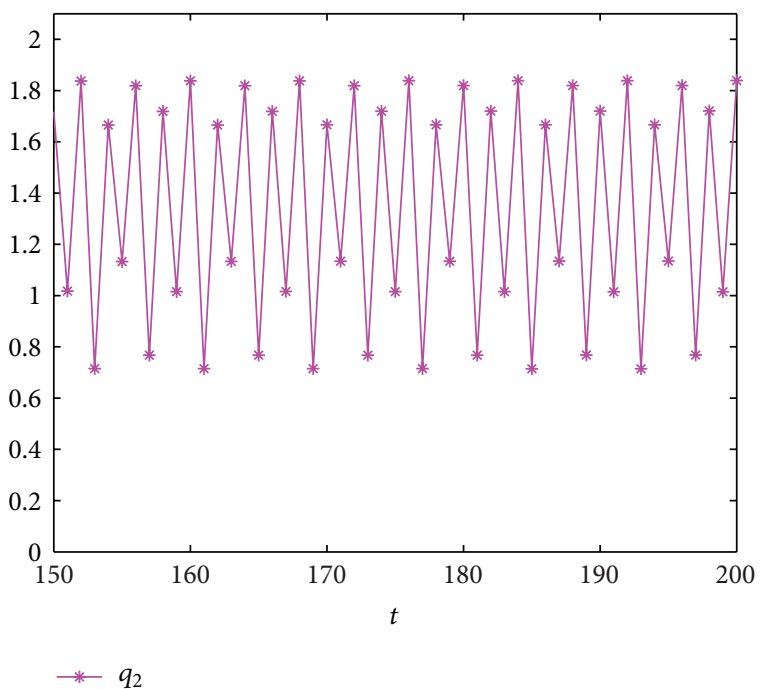

FIGURE 13: The wave plots of the output $q_{2}$ with the change of time when $\alpha_{1}=1.02$ and $\alpha_{2}=1.46, \theta=0.394$.

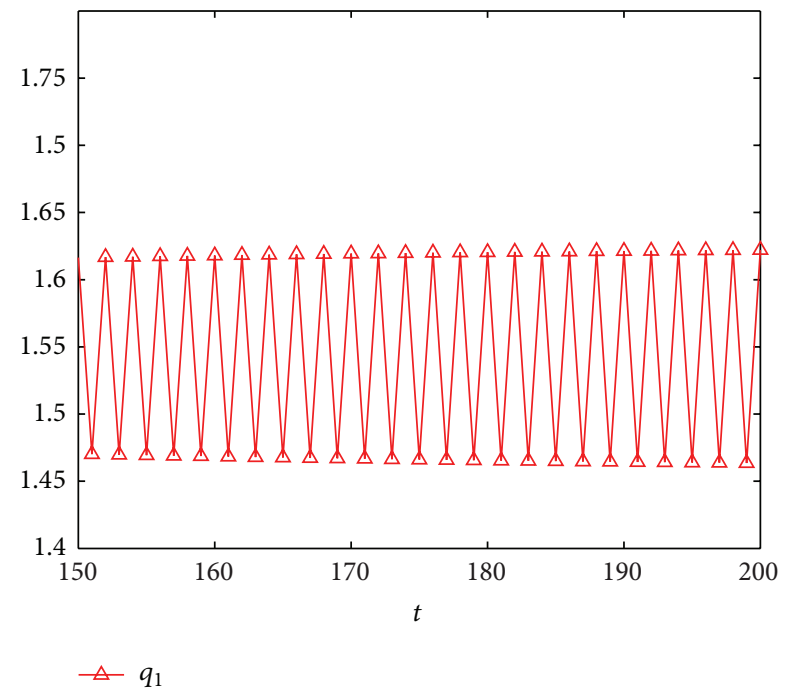

FIGURE 14: The wave plots of the output $q_{1}$ with the change of time when $\alpha_{1}=1.02, \alpha_{2}=1.46$ and $\theta=0.664$.

the chaotic motion has self-similar structure, which is an important difference between the chaotic motion and the stochastic motion. The fractal dimension of system (6) is $d=$ $2-\left(\lambda_{1} / \lambda_{2}\right) \approx 1.931876$. The fractal dimension also reflects the space density of the chaotic attractor. The larger the dimension of the chaotic attractor is, the larger the occupied space is. Thus, the structure of the chaotic attractor is more compact, and the system is more complex and vice versa. The fractal dimension of the 2D discrete system (6) is more than 1.5 , so the occupied space is broad, and the structure is tight, which can be seen in Figure 5 .

\section{Chaos Control}

We can see that system (6) becomes unstable and eventually falls into chaos if the output adjustment speed parameter

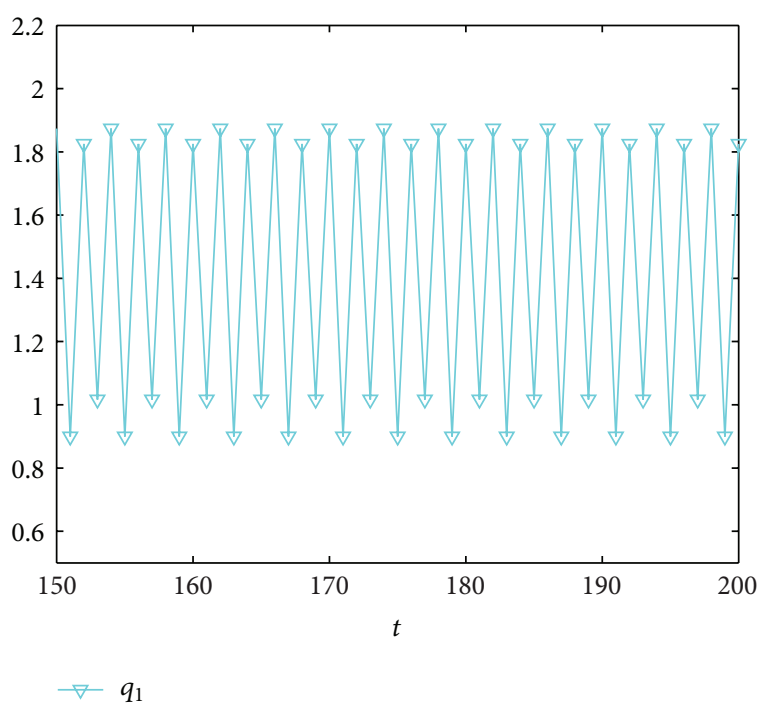

FIGURE 15: The wave plots of the output $q_{1}$ with the change of time when $\alpha_{1}=1.02, \alpha_{2}=1.46$ and $\theta=0.812$.

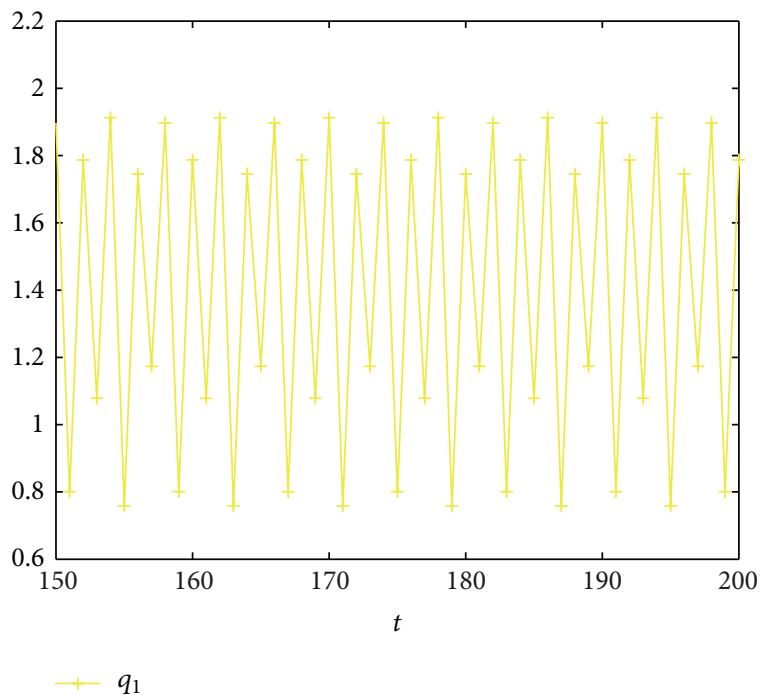

FIGURE 16: The wave plots of the output $q_{1}$ with the change of time when $\alpha_{1}=1.02, \alpha_{2}=1.46$ and $\theta=0.843$.

$\alpha_{i}(i=1,2)$ exceeds a critical value. Both the two renewable resource companies will be harmed and the renewable resource market will become irregular when chaos occurs. Therefore, no one is able to make good strategies and decide reasonable output. To avert risk, it is a good idea for the duopoly renewable resource firms to stay at Nash equilibrium state.

In this part, the parameter adjustment method is used to control the chaos of system (6). The system which is under controlled is as follows:

$q_{1}(t+1)$

$=(1-v)$ 


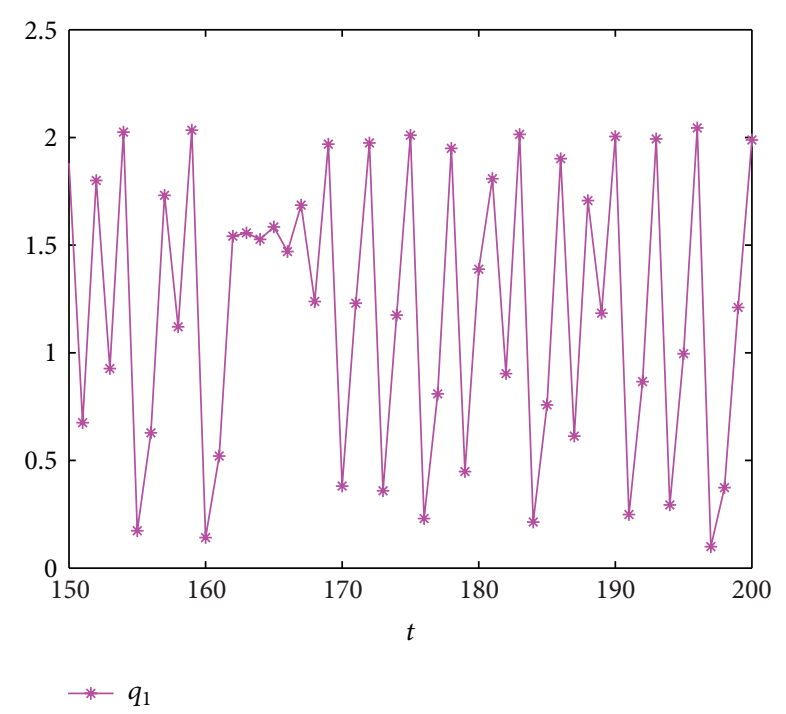

FIgURE 17: The wave plots of the output $q_{1}$ with the change of time when $\alpha_{1}=1.02, \alpha_{2}=1.46$ and $\theta=0.976$.

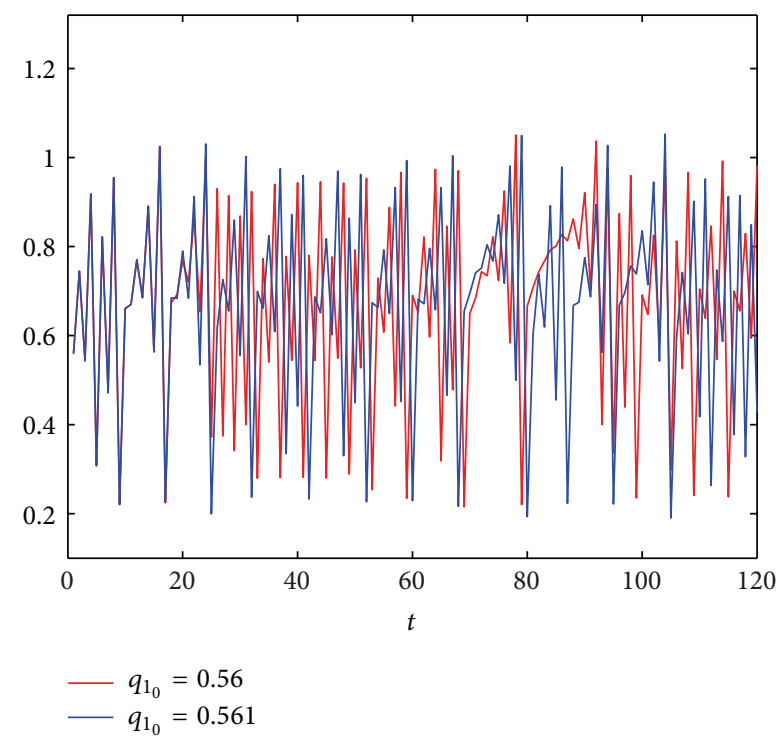

FIgURE 18: For $\alpha_{1}=2.05, \alpha_{2}=1.46$ and $\theta=0.5$, and the initial conditions which are $(0.56,0.92)$ and $(0.561,0.92)$, the history diagram with the change of time about the output $q_{1}$.

$$
\begin{aligned}
\times\left\{q_{1}(t)+\right. & \alpha_{1} q_{1}(t) \\
\times & {\left[\theta\left(a-b Q_{T}(t)-b q_{1}-\frac{2 d_{1} q_{1}(t)}{Q_{T}(t)}+\frac{d_{1} q_{1}^{2}(t)}{Q_{T}^{2}(t)}\right)\right.} \\
& \left.\left.+(1-\theta)\left(-b q_{2}(t)+\frac{d_{2} q_{2}^{2}(t)}{Q_{T}^{2}(t)}\right)\right]\right\}+v q_{1}(t),
\end{aligned}
$$

$q_{2}(t+1)$

$=(1-v)$

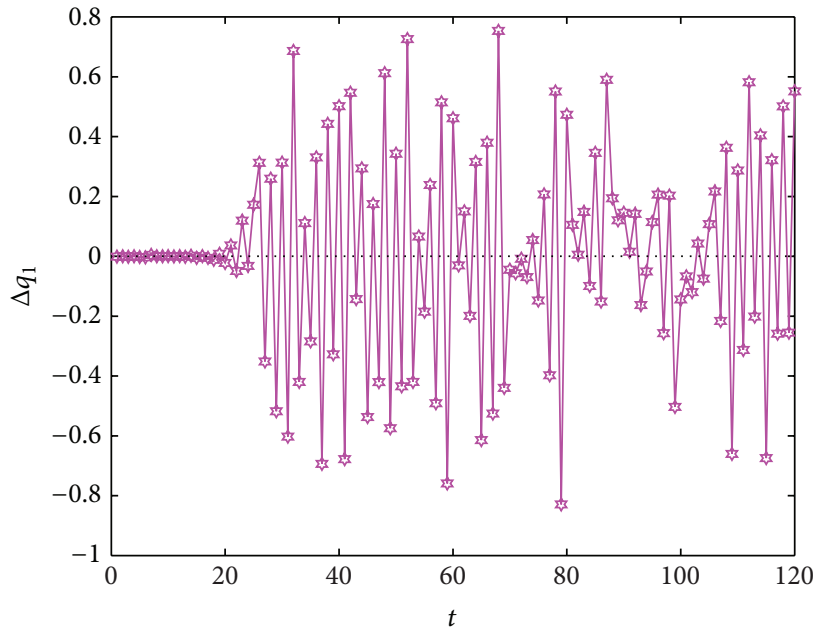

FIgURE 19: For $\alpha_{1}=2.05, \alpha_{2}=1.46$ and $\theta=0.5$, and the initial conditions which are $(0.56,0.92)$ and $(0.561,0.92)$, the difference with the change of time about the output $q_{1}$.

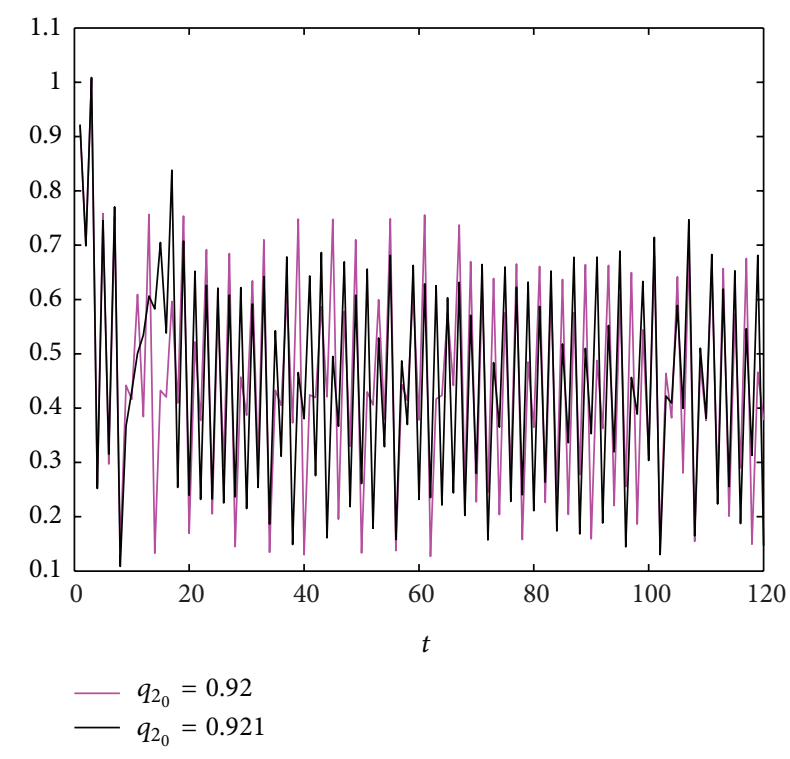

FIgURE 20: For $\alpha_{1}=1.32, \alpha_{2}=2.471$ and $\theta=0.5$, and the initial conditions which are $(0.76,0.92)$ and $(0.76,0.921)$, the history diagram with the change of time about the output $q_{2}$.

$$
\begin{aligned}
& \times\left\{q_{2}(t)+\alpha_{2} q_{2}(t)\right. \\
& \times\left[(1-\theta)\left(a-b Q_{T}(t)-b q_{2}-\frac{2 d_{2} q_{2}(t)}{Q_{T}(t)}+\frac{d_{2} q_{2}^{2}(t)}{Q_{T}^{2}(t)}\right)\right. \\
& \left.\left.\quad+\theta\left(-b q_{1}(t)+\frac{d_{1} q_{1}^{2}(t)}{Q_{T}^{2}(t)}\right)\right]\right\}+v q_{2}(t),
\end{aligned}
$$

where $0<v<1$ is the adjustment parameter and other parameters are the same as above. 


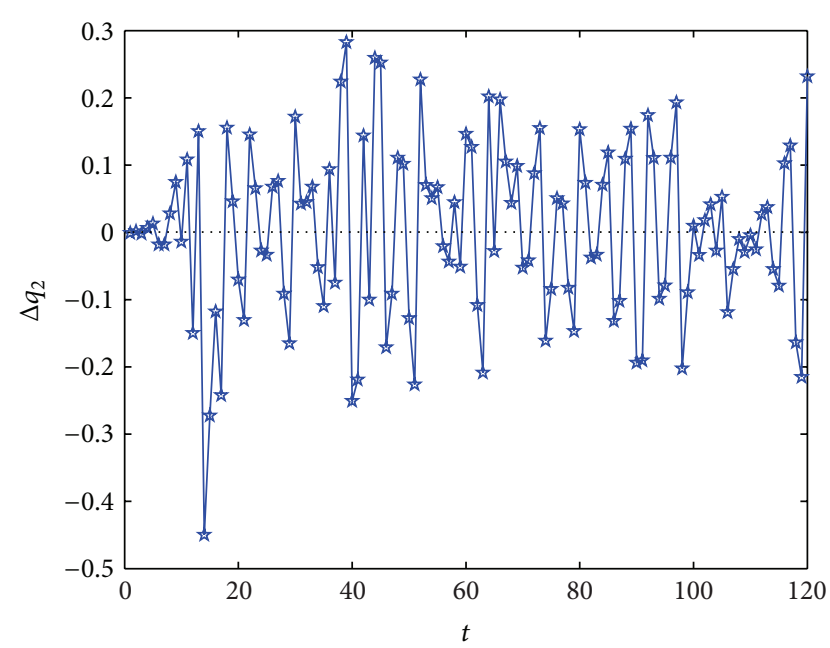

FIGURE 21: For $\alpha_{1}=1.32, \alpha_{2}=2.471$ and $\theta=0.5$, and the initial conditions which are $(0.76,0.92)$ and $(0.76,0.921)$, the difference with the change of time about the output $q_{2}$.

It can be seen from the bifurcation diagram in Figure 22 and the corresponding Lyapunov exponents path in Figure 23 that, for $\alpha_{2}=1.46$ and $v=0.157$, controlled system (13) stabilized at Nash equilibrium point for $v>0.2867$. It demonstrates that chaos control of the system (6) can be realized by a small parameter adjustment.

When $\alpha_{2}=1.46$ and $v=0.157$, the stable region of $\alpha$ expands from the original 2-cycle bifurcation point $\alpha_{1}=$ 1.16546 in Figure 2 to $\alpha_{1}=1.6091$ in Figure 24, which implies that once system is under control, chaos is delayed or eliminated completely.

The purpose of system control is to take effective measures to regulate market behaviors for avoiding the occurrence of chaos. The system can regain equilibrium state by the parameter adjustment method when the system (6) is in a chaotic state, and the bifurcation and chaos behaviors of the system can be delayed or eliminated. The parameter adjustment method can ensure that the renewable resource market develop orderly, and the renewable resource firms compete rationally and healthily.

\section{Model of Evolutionary Game}

Assume that all the firms in the market take the strategy of team combination. Every combination is based on the last stage, which is similar to the view of biological evolution [20]. Thus, we analyze the game process using the team combination strategy under the theory of evolutionary game. The types of free combination which $p_{1}$ can take are $\left(p_{1}, p_{1}\right)$, $\left(p_{1}, p_{2}\right)$, and $\left(p_{1}, p_{3}\right)$; the probability of free combination for $\left(p_{1}, p_{2}\right)$ is $\widetilde{u_{12}}$; the probability of free combination for $\left(p_{1}, p_{3}\right)$ is $\widetilde{u_{13}}$. The types of free combination which $p_{2}$ can take are $\left(p_{2}, p_{2}\right),\left(p_{2}, p_{1}\right)$, and $\left(p_{2}, p_{3}\right)$; the probability of free combination for $\left(p_{2}, p_{1}\right)$ is $\widetilde{u_{21}}$; the probability of free combination

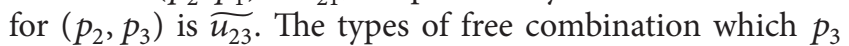
can take are $\left(p_{3}, p_{3}\right),\left(p_{3}, p_{1}\right)$, and $\left(p_{3}, p_{2}\right)$; the probability

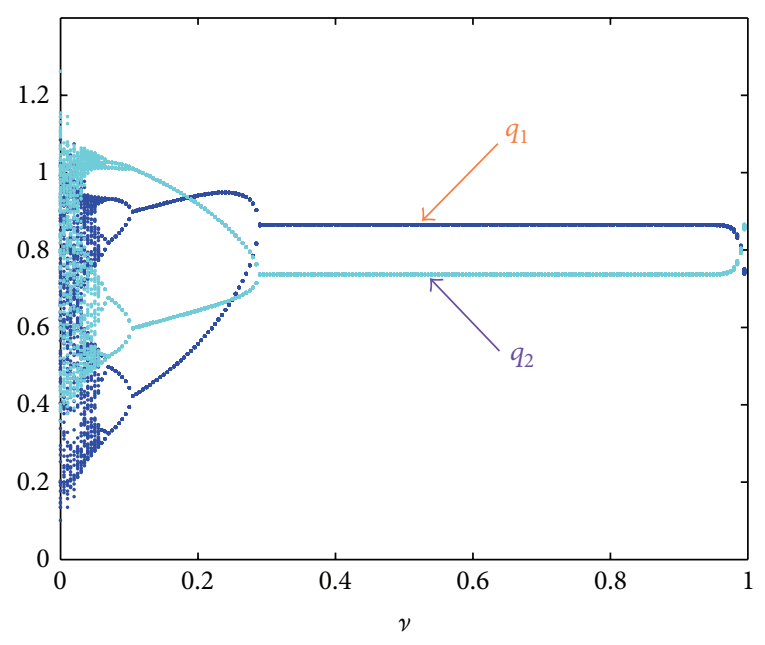

FIGURE 22: Bifurcation diagram of system (13) with $v \in(0,1], \alpha_{1}=$ 2.13 and $\alpha_{2}=1.46$.

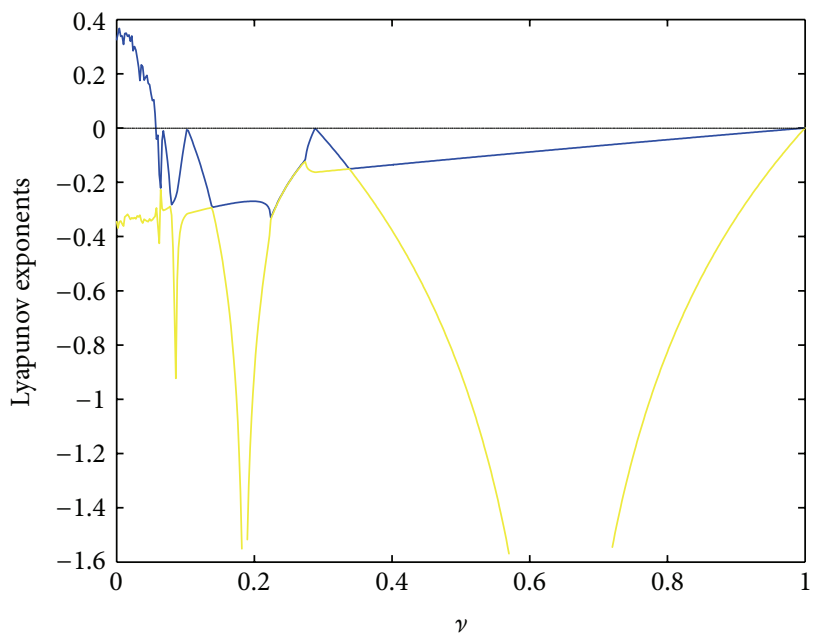

FIGURE 23: The corresponding Lyapunov exponents with $v \in(0,1]$, $\alpha_{1}=2.13$ and $\alpha_{2}=1.46$.

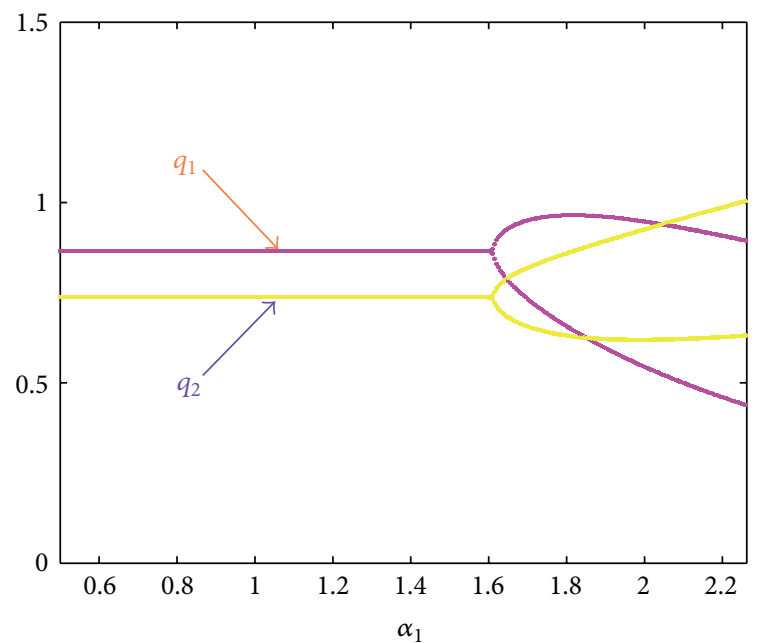

Figure 24: Bifurcation diagram of system (13) with $\alpha_{1} \epsilon$ $(0.5,2.2622]$, and $\alpha_{2}=1.46$ and $\nu=0.157$. 


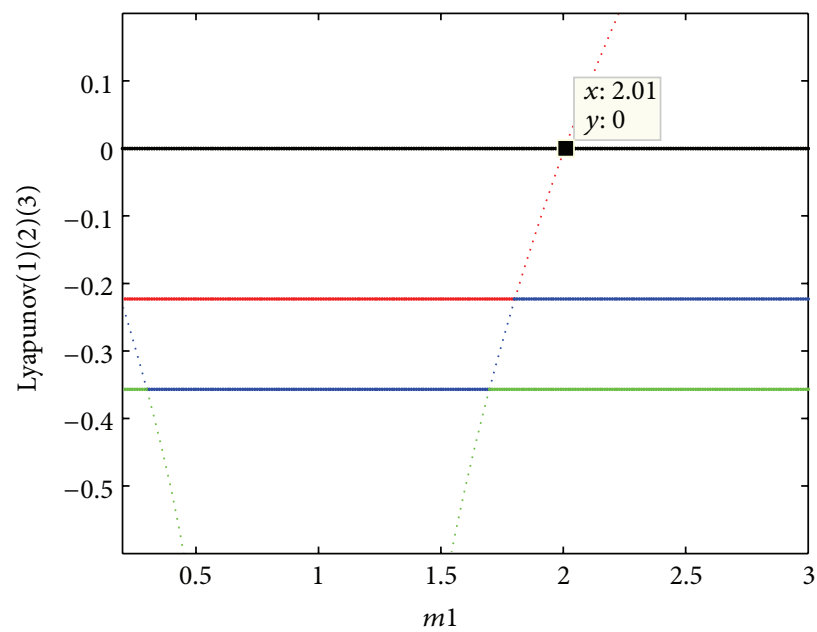

(a) The Lyapunov exponents with the varies of $m 1$, when $x=0.45, y=$ $0.2, z=0.3, a=0.1, b=0.4, c=0.62, m 2=0.3$, and $m 3=0.2$

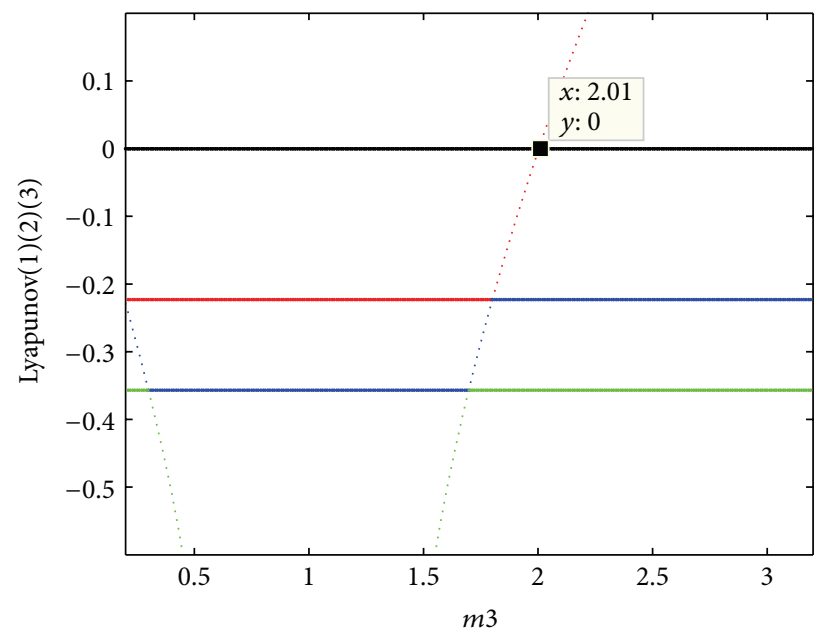

(c) The Lyapunov exponents with the varies of $m 3$, when $x=0.45, y=$ $0.2, z=0.3, a=0.1, b=0.4, c=0.62, m 1=0.2$, and $m 2=0.3$

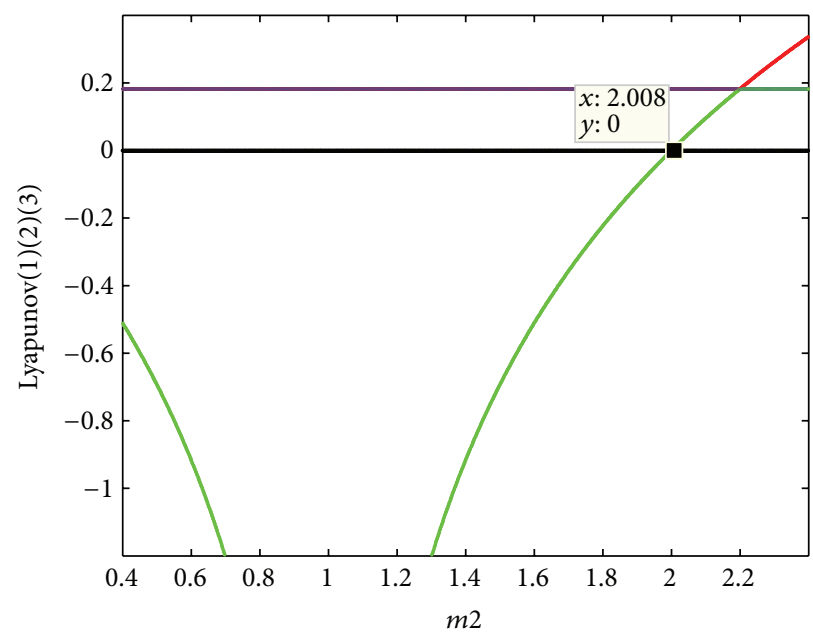

(e) The Lyapunov exponents with the varies of $m 2$, when $x=0.45, y=$ $0.2, z=0.3, a=0.1, b=0.4, c=0.62, m 1=2.2$, and $m 3=2.2$

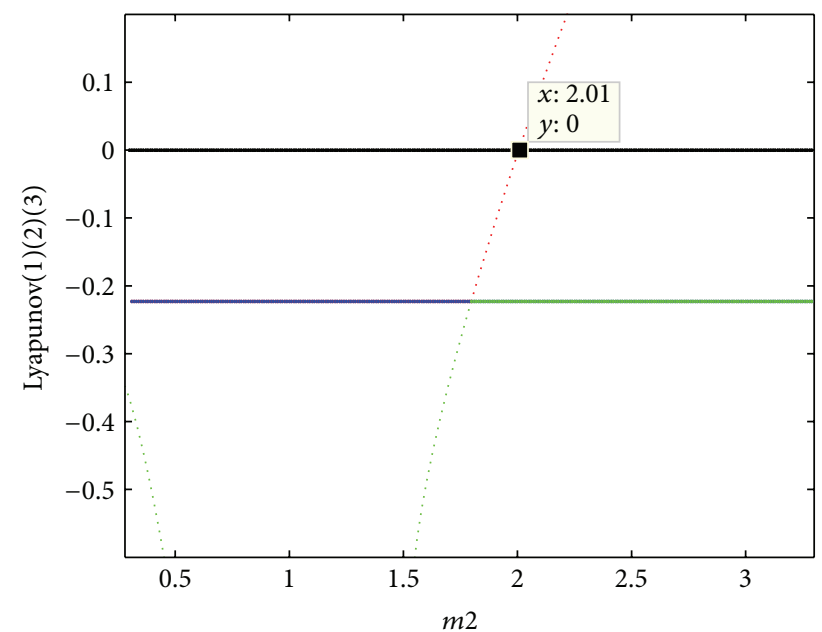

(b) The Lyapunov exponents with the varies of $m 2$, when $x=0.45, y=$ $0.2, z=0.3, a=0.1, b=0.4, c=0.62, m 1=0.2$, and $m 3=0.2$

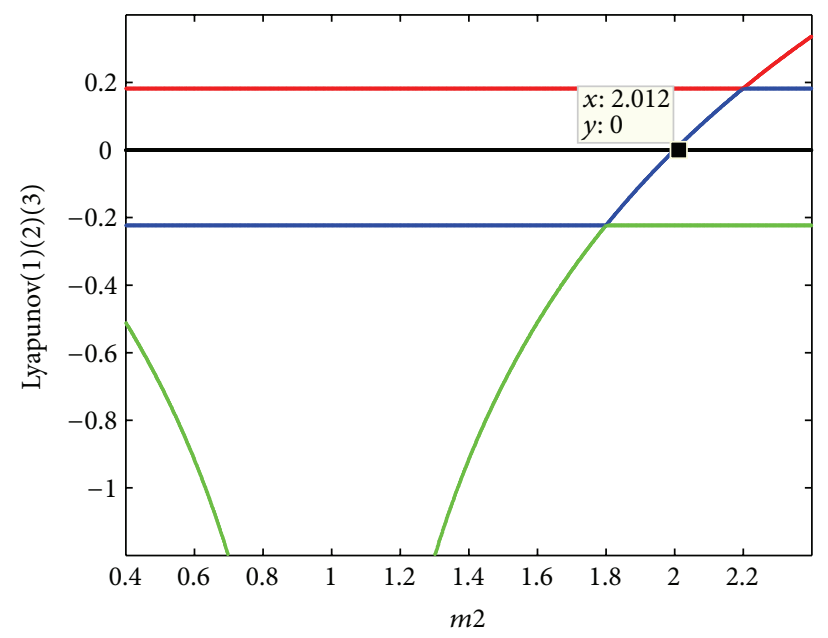

(d) The Lyapunov exponents with the varies of $m 2$, when $x=0.45, y=$ $0.2, z=0.3, a=0.1, b=0.4, c=0.62, m 1=2.2$, and $m 3=0.2$

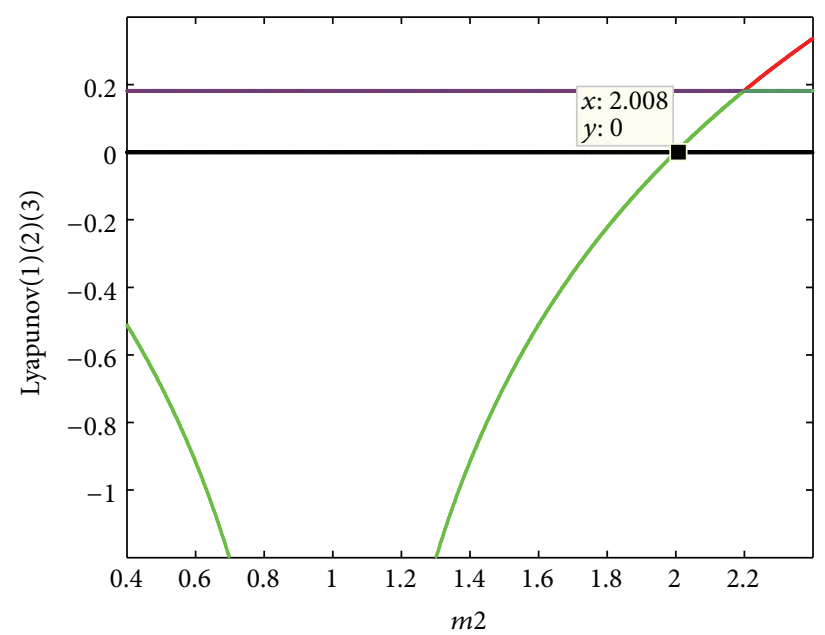

(f) The Lyapunov exponents with the varies of $m 2$, when $x=0.85, y=$ $0.62, z=0.38, a=0.1, b=0.4, c=0.62, m 1=2.2$, and $m 3=2.2$

FIGURE 25: The Lyapunov exponents with the varies of parameters $a(a \in[1,2.6])$ and $b(b \in[12,22])$ when the step length is 0.001 . 


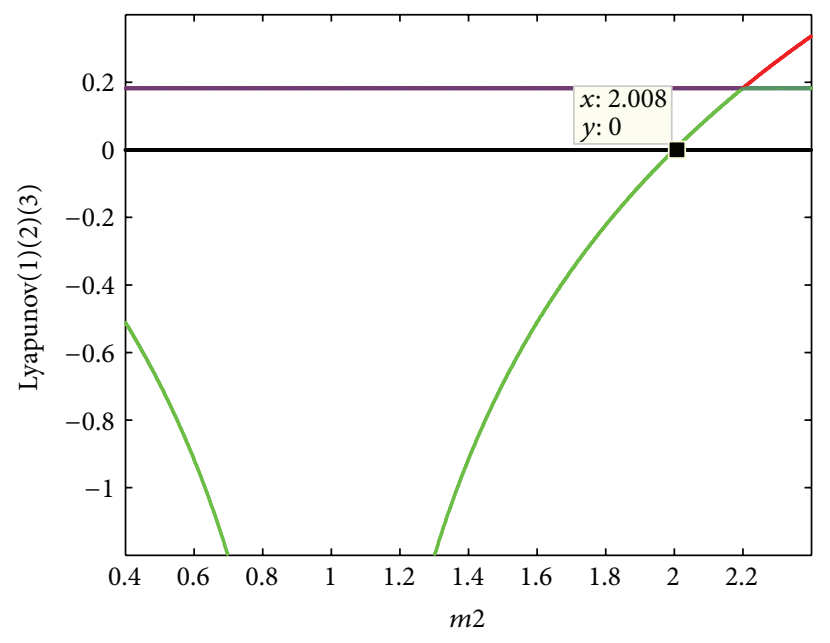

(a) The Lyapunov exponents with the varies of $m 2$, when $x=0.85, y=$ $0.62, z=0.38, a=0.3, b=0.9, c=0.55, m 1=2.2$, and $m 3=2.2$

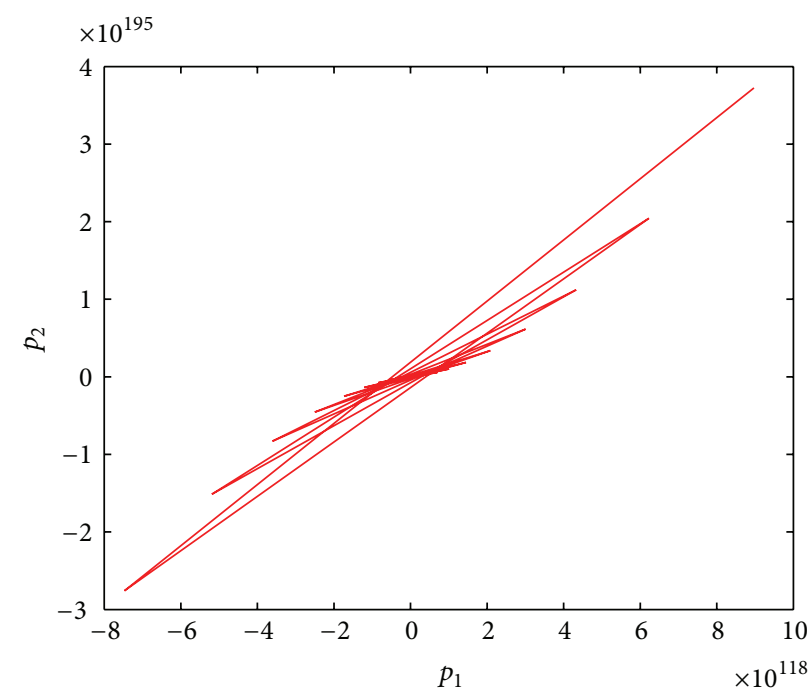

(c) Projection onto the $p_{1}-p_{2}$ plane of the attractors

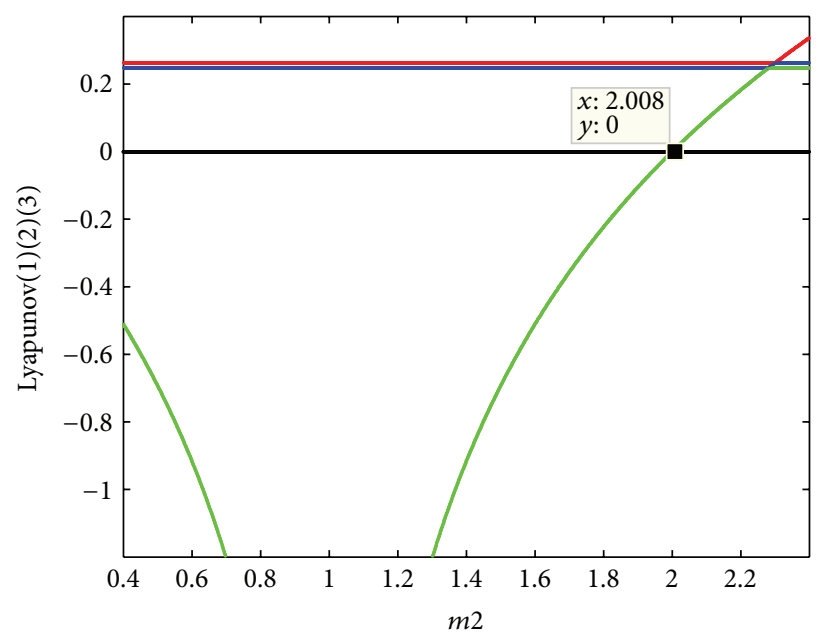

(b) The Lyapunov exponents with the varies of $m 2$, when $x=0.85, y=$ $0.62, z=0.38, a=0.3, b=0.9, c=0.55, m 1=2.28$, and $m 3=2.3$

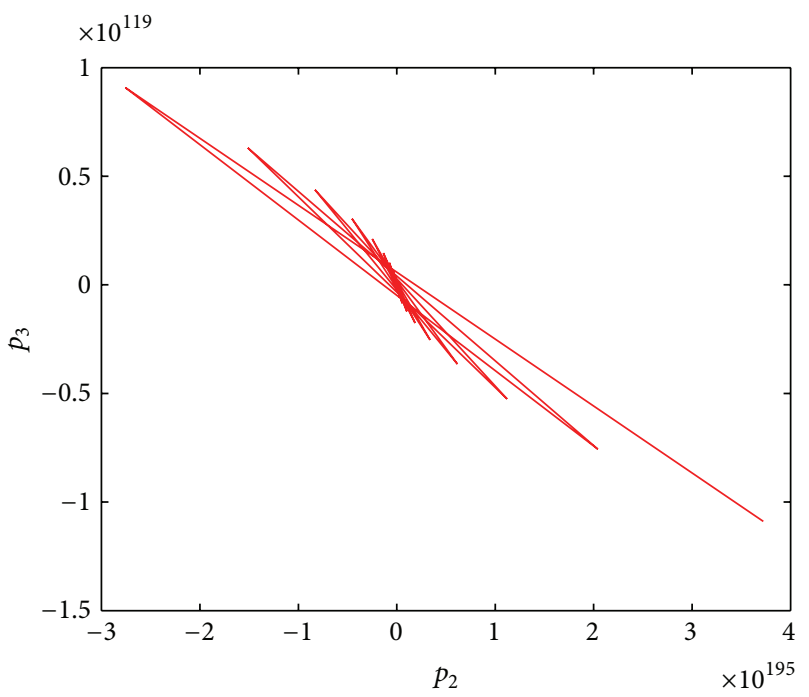

(d) Projection onto the $p_{2}-p_{3}$ plane of the attractors

FIgURE 26: The Lyapunov exponents with the varies of parameters $a(a \in[1,2.6])$ and $b(b \in[12,22])$ when the step length is 0.001 .

of free combination for $\left(p_{3}, p_{1}\right)$ is $\widetilde{u_{31}}$; the probability of free combination for $\left(p_{3}, p_{2}\right)$ is $\widetilde{u_{32}}$. Consider

$$
\begin{aligned}
& u=\left(\begin{array}{lll}
2 & 6 & 7 \\
4 & 8 & 8 \\
3 & 2 & 5
\end{array}\right), \\
& \widetilde{u_{i j}}=\frac{u_{i j}}{u_{i j}+u_{j i}} .
\end{aligned}
$$

We make $\widetilde{u_{12}}=a, \widetilde{u_{13}}=b$, and $\widetilde{u_{23}}=c$; thus, $\widetilde{u_{21}}=1-a$, $\widetilde{u_{31}}=1-b$, and $\widetilde{u_{32}}=1-c$, and the model can be written:

$$
\begin{gathered}
p_{1}(i+1)=p_{1}^{2}(i)+2 a p_{1}(i) p_{2}(i)+2 b p_{1}(i) p_{3}(i) \\
p_{2}(i+1)=p_{2}^{2}(i)+2(1-a) p_{1}(i) p_{2}(i)+2 c p_{2}(i) p_{3}(i)
\end{gathered}
$$

$$
\begin{aligned}
p_{3}(i+1)= & p_{3}^{2}(i)+2(1-b) p_{1}(i) p_{3}(i) \\
& +2(1-c) p_{2}(i) p_{3}(i),
\end{aligned}
$$

Solve the equation; then we can obtain the equilibrium points:

$$
\begin{aligned}
& p_{1}=\frac{(2 c-1)}{(2 a-2 b+2 c-1)}, \\
& p_{2}=-\frac{(2 b-1)}{(2 a-2 b+2 c-1)} \\
& p_{3}=\frac{(2 a-1)}{(2 a-2 b+2 c-1)}
\end{aligned}
$$

The dynamic equation can be written as follows:

$$
p_{1}(i+1)=p_{1}(i)(1-m 1)+m_{1} \frac{(2 c-1)}{(2 a-2 b+2 c-1)},
$$




$$
\begin{aligned}
& p_{2}(i+1)=p_{2}(i)(1-m 2)-m_{2} \frac{(2 b-1)}{(2 a-2 b+2 c-1)}, \\
& p_{3}(i+1)=p_{3}(i)(1-m 3)+m_{3} \frac{(2 a-1)}{(2 a-2 b+2 c-1)},
\end{aligned}
$$

where $m_{i}(i=1,2,3)$ is the adjustment speed parameter.

6.1. Lyapunov Exponents and Chaotic Attractors. As it can be seen, Figure 25(a) is the Lyapunov exponents with the varies of $m 1$, when $x=0.45, y=0.2, z=0.3, a=0.1, b=0.4$, $c=0.62, m 2=0.3$, and $m 3=0.2$; Figure $25(\mathrm{~b})$ is the Lyapunov exponents with the varies of $m 2$, when $x=0.45$, $y=0.2, z=0.3, a=0.1, b=0.4, c=0.62, m 1=0.2$, and $m 3=0.2$; Figure 25(c) is the Lyapunov exponents with the varies of $m 3$, when $x=0.45, y=0.2, z=0.3, a=0.1$, $b=0.4, c=0.62, m 1=0.2$, and $m 2=0.3$; Figure $25(\mathrm{~d})$ is the Lyapunov exponents with the varies of $m 2$, when $x=0.45$, $y=0.2, z=0.3, a=0.1, b=0.4, c=0.62, m 1=2.2$, and $m 3=0.2$; Figure 25(e) is the Lyapunov exponents with the varies of $m 2$, when $x=0.45, y=0.2, z=0.3, a=0.1$, $b=0.4, c=0.62, m 1=2.2$, and $m 3=2.2$; Figure $25(\mathrm{f})$ is the Lyapunov exponents with the varies of $m 2$, when $x=$ $0.85, y=0.62, z=0.38, a=0.1, b=0.4, c=0.62$, $m 1=2.2$, and $m 3=2.2$. The evolutionary game model is a special nonlinear dynamic model. In the process of evolution, when the adjust parameter is 2.01 , the system enters a chaotic state. According to the numerical simulation, it shows that the state has nothing to do with the initial probability and the coefficient of the dynamic equation. Whatever initial values and parameters change into, it is at the point of 2.01 that the first largest Lyapunov index changes into zero, and the system becomes chaotic, the second and third largest lyapunov index changing into zero. Thus, the 2.01 is a key point, which is a border of a bifurcation point from the steady state to chaos in the process of evolutionary game. In order to prevent the system enters a chaotic state, it requires us to control the parameters. When the adjustment speed parameter changes too fast, the system enters a chaotic or hyperchaotic state. It is known to us that the direction of evolutionary game will be decided by the initial value. Whether the system goes into a chaotic or hyperchaotic state, it has no relation with the initial values and the direction of the evolution, but the rate of evolution. That is to say, there are three sides in the process of evolution. One part will enter a state of chaos when the adjust parameter is 2.01 and the first largest lyapunov exponent is zero; thus the system becomes chaotic; when the second largest lyapunov exponent is zero and the adjust parameter is 2.01, two positive lyapunov exponents exist and the system goes into hyperchaotic state. When the evolutionary rate increases to 2.01 for the third party, the system appears there are three positive lyapunov exponents, and system enters a state of trichaos. Figure 26(a) is the Lyapunov exponents with the varies of $m 2$, when $x=0.85, y=0.62, z=0.38, a=0.3$, $b=0.9, c=0.55, m 1=2.2$, and $m 3=2.2$; Figure $26(\mathrm{~b})$ is the Lyapunov exponents with the varies of $m 2$, when $x=0.85$, $y=0.62, z=0.38, a=0.3, b=0.9, c=0.55, m 1=2.28$, and $m 3=2.3$; we obtain that when the adjust rate parameter $m 2$ changes, the point of the third lyapunov exponent becoming zero is still at 2.01; Figure 26(c) is the projection onto the $p_{1}$ $p_{2}$ plane of the attractors and Figure 26(d) is the projection onto the $p_{2}-p_{3}$ plane of the attractors when $x=0.45, y=0.2$, $z=0.3, a=0.1, b=0.4, c=0.62, m 1=2.3, m 2=2.5$, and $m 3=2.3$.

\section{Conclusions}

A new dynamics of a nonlinear duopoly game with team players in exploitation of a renewable resource is built up in this paper. The stability of the Nash equilibrium point and the bifurcation and chaotic behaviors of the dynamic system are studied. It is found that bifurcation and chaos occur with the increasing of the output adjustment speed parameters $\alpha_{i}(i=1,2)$ and the change of weight parameter $\theta$. The weight parameter $\theta$ not only affects the stability of system (6), but also changes the Nash equilibrium point of the system. The oligopoly market may become unstable and even fall into chaos while the output adjustment speed parameters are out of the stable region. Furthermore, the chaos can be delayed or eliminated by the parameter adjustment method.

A model of evolutionary game is constructed in this paper, and the dynamic behaviors are discussed. We can obtain some useful results indicating that evolutionary game model is a special dynamic system, because it is at the point of 2.01 that every lyapunov index changes into chaos, no matter how the parameters change. Thus, it will be helpful for us to adopt some measures in the process of game.

It has a very theoretical and practical significance to research the complexity of new nonlinear dynamical system. As the traditional nonrenewable energy is being depleted, the development of renewable resource is an inevitable choice. This paper also shows a guidance for the renewable resource firms to make strategies of their output and exploit the renewable resource and is helpful for the government to formulate relevant policies to manage the renewable resource market.

\section{Conflict of Interests}

The authors declare that there is no conflict of interests regarding the publication of this paper.

\section{Acknowledgments}

The authors would like to seize this opportunity to thank the National Nature Science Foundation of China (Grant no. 61273231). This work was supported by Doctoral Fund of Ministry of Education of China (Grant no. 20130032110073).

\section{References}

[1] T. Puu, "The chaotic duopolists revisited," Journal of Economic Behavior and Organization, vol. 33, no. 3-4, pp. 385-394, 1998.

[2] H. N. Agiza, "Explicit stability zones for Cournot game with 3 and 4 competitors," Chaos, Solitons and Fractals, vol. 9, no. 12, pp. 1955-1966, 1998. 
[3] H. N. Agiza, "On the analysis of stability, bifurcation, chaos and chaos control of Kopel map," Chaos, Solitons and Fractals, vol. 10, no. 11, pp. 1909-1916, 1999.

[4] H. N. Agiza and A. A. Elsadany, "Chaotic dynamics in nonlinear duopoly game with heterogeneous players," Applied Mathematics and Computation, vol. 149, no. 3, pp. 843-860, 2004.

[5] T. Dubiel-Teleszynski, "Nonlinear dynamics in a heterogeneous duopoly game with adjusting players and diseconomies of scale," Communications in Nonlinear Science and Numerical Simulation, vol. 16, no. 1, pp. 296-308, 2011.

[6] D. Dragone, L. Lambertini, G. Leitmann, and A. Palestini, "A stochastic optimal control model of pollution abatement," Nonlinear Dynamics and Systems Theory, vol. 10, no. 2, pp. 117-124, 2010.

[7] A. A. Elsadany, "Dynamics of a delayed duopoly game with bounded rationality," Mathematical and Computer Modelling, vol. 52, no. 9-10, pp. 1479-1489, 2010.

[8] J. Ma and X. Pu, "Complex dynamics in nonlinear triopoly market with different expectations," Discrete Dynamics in Nature and Society, vol. 2011, Article ID 902014, 12 pages, 2011.

[9] J. Ma and Y. Yang, "Hyperchaos numerical simulation and control in a 4D hyperchaotic system," Discrete Dynamics in Nature and Society, vol. 2013, Article ID 980578, 16 pages, 2013.

[10] X. Xu, Z. Chen, G. Si, X. Hu, Y. Jiang, and X. Xu, "The chaotic dynamics of the social behavior selection networks in crowd simulation," Nonlinear Dynamics, vol. 64, no. 1-2, pp. 117-126, 2011.

[11] J. Ding, Q. Mei, and H. Yao, "Dynamics and adaptive control of a duopoly advertising model based on heterogeneous expectations," Nonlinear Dynamics, vol. 67, no. 1, pp. 129-138, 2012.

[12] Y. Liu, X. Chen, L. Zhang, F. Tao, and L. Wang, "Does migration cost influence cooperation among success-driven individuals," Chaos Solitons Fractals, vol. 45, pp. 1301-1308, 2012.

[13] H. Li, Q. Dai, H. Cheng, and J. Yang, "Cooperation in an evolutionary prisoner's dilemma game with probabilistic strategies," Chaos Solitons Fractals, vol. 45, pp. 1397-1403, 2012.

[14] E. Ahmed and A. S. Hegazi, "On dynamical multi-team and signaling games," Applied Mathematics and Computation, vol. 172, no. 1, pp. 524-530, 2006.

[15] E. Ahmed, M. F. Elettreby, and A. S. Hegazi, "On Puu's incomplete information formulation for the standard and multi-team Bertrand game," Chaos, Solitons \& Fractals, vol. 30, no. 5, pp. 1180-1184, 2006.

[16] E. Ahmed, M. F. Elettreby, and A. S. Hegazi, "On quantum team games," International Journal of Theoretical Physics, vol. 45, no. 5, pp. 907-913, 2006.

[17] M. F. Elettreby and S. Z. Hassan, "Dynamical multi-team Cournot game," Chaos, Solitons \& Fractals, vol. 27, no. 3, pp. 666-672, 2006.

[18] M. F. Elettreby and M. Mansour, "On Cournot dynamic multiteam game using incomplete information dynamical system," Applied Mathematics and Computation, vol. 218, no. 21, pp. 10691-10696, 2012.

[19] S. S. Asker, "On dynamical multi-team Cournot game in exploitation of a renewable resource," Chaos, Solitons and Fractals, vol. 32, no. 1, pp. 264-268, 2007.

[20] C. Veller and V. Rajpaul, "Purely competitive evolutionary dynamics for games," Physical Review E, vol. 86, no. 4, Article ID 041907, 2012.

[21] L. Edelstein-Kashet, Mathematical Models in Biology, Random House, New York, NY, USA, 1992.
[22] J. L. Kaplan and J. A. Yorke, "Preturbulence: a regime observed in a fluid flow model of Lorenz," Communications in Mathematical Physics, vol. 67, no. 2, pp. 93-108, 1979.

[23] H. Wang and J. Ma, "Complexity analysis of a Cournot-Bertrand duopoly game model with limited information," Discrete Dynamics in Nature and Society, vol. 2013, Article ID 287371, 6 pages, 2013.

[24] J. Ma and H. Tu, "Complexity of a duopoly game in the electricity market with delayed bounded rationality," Discrete Dynamics in Nature and Society, vol. 2012, Article ID 698270, 13 pages, 2012. 


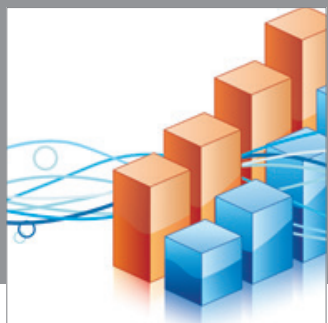

Advances in

Operations Research

mansans

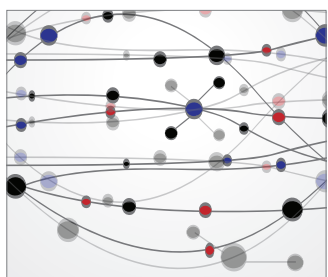

The Scientific World Journal
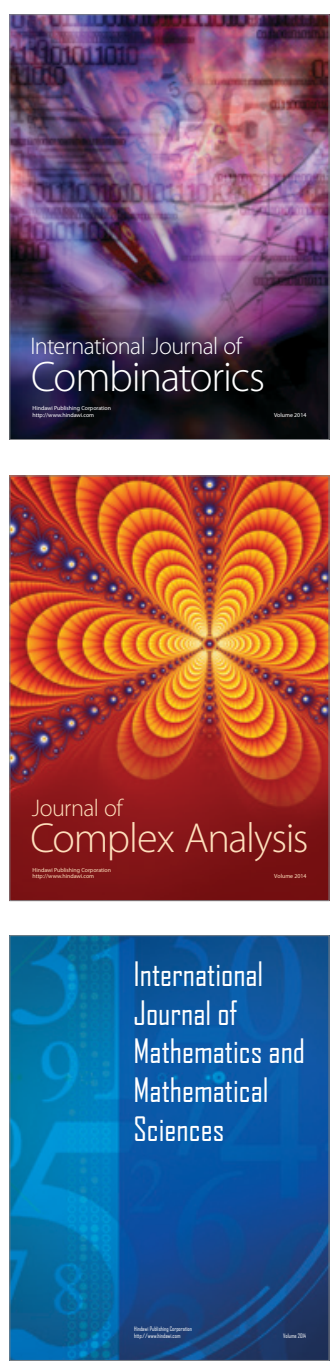
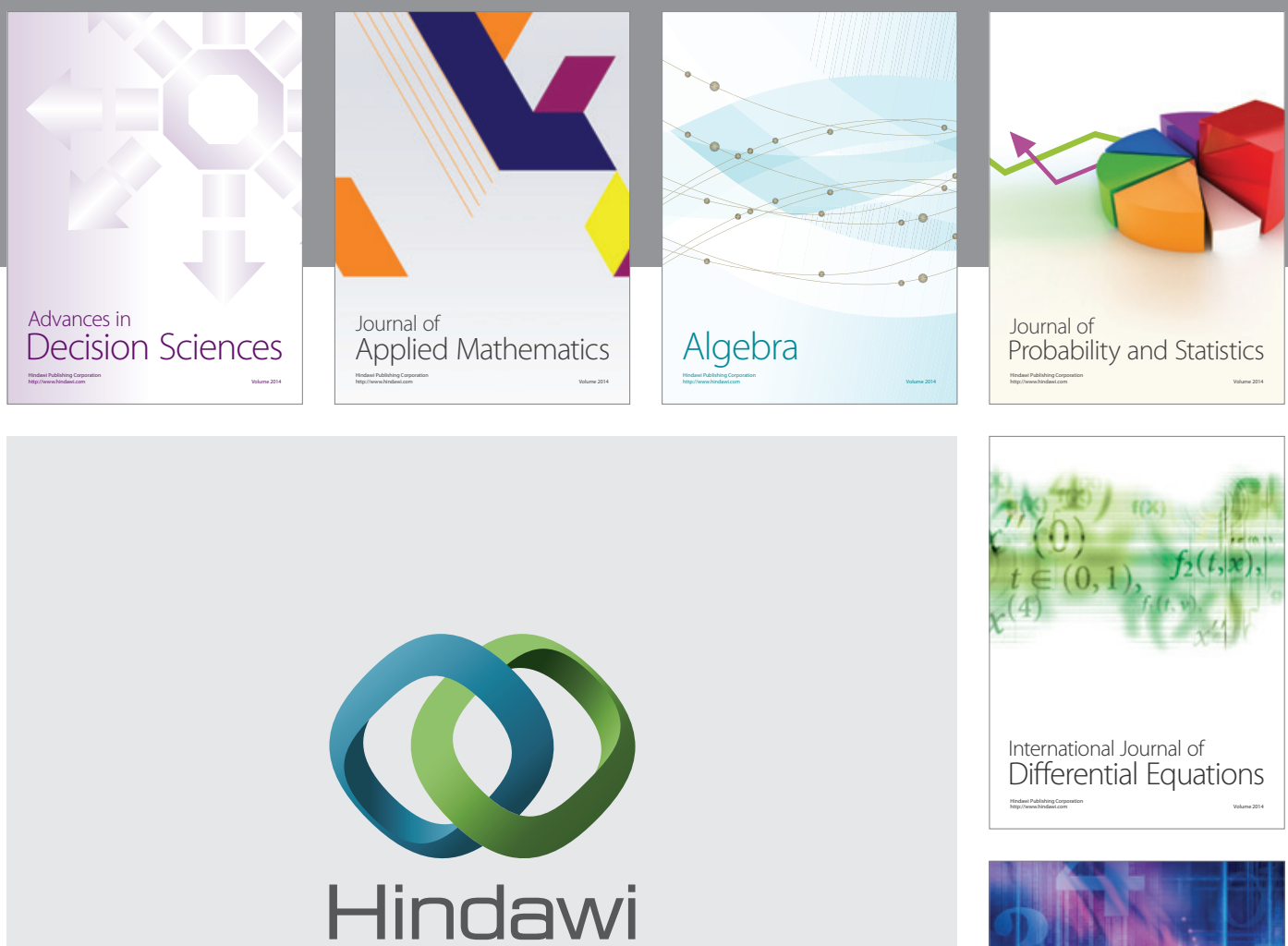

Submit your manuscripts at http://www.hindawi.com
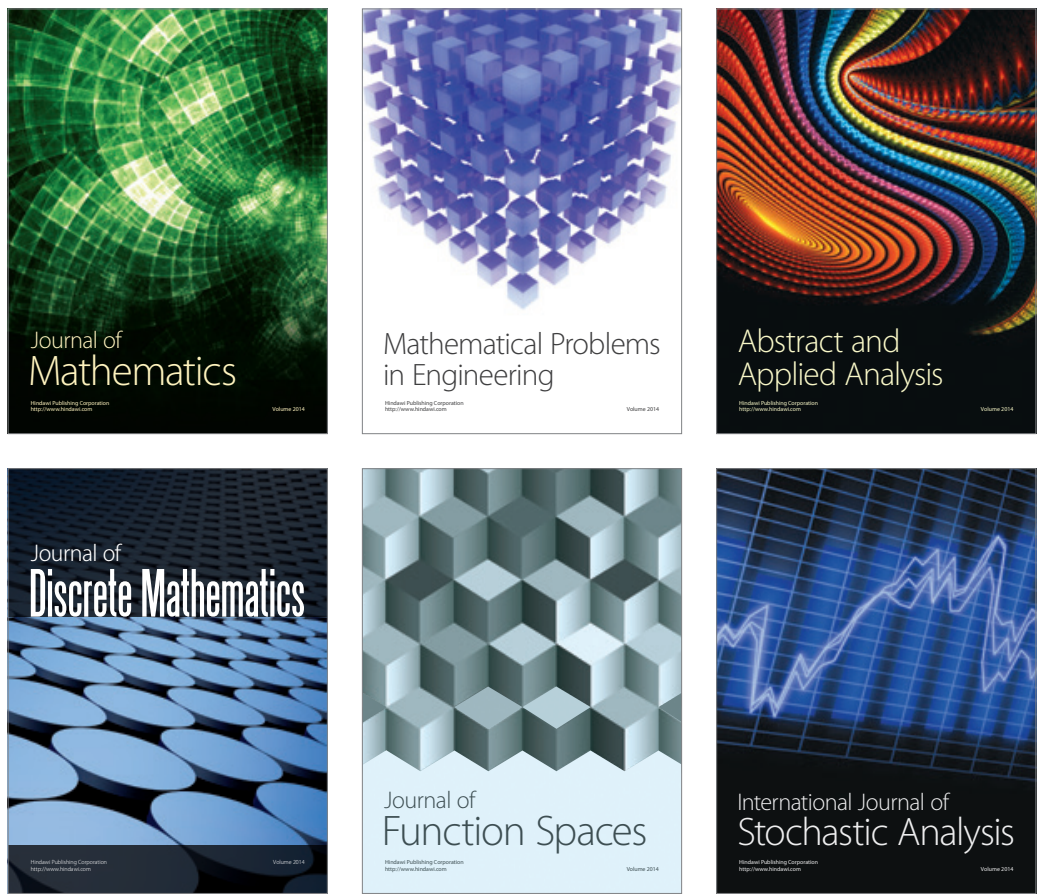

Journal of

Function Spaces

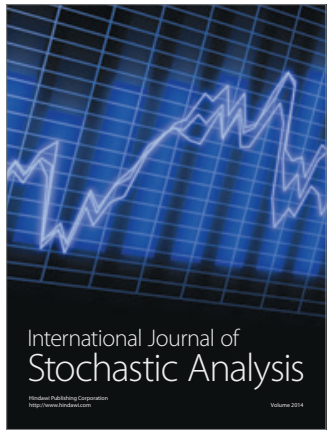

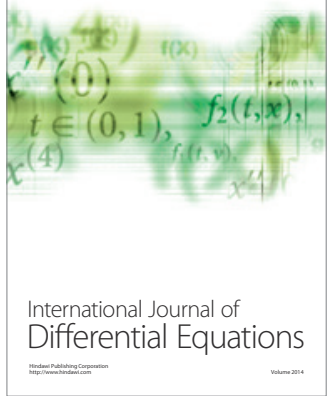
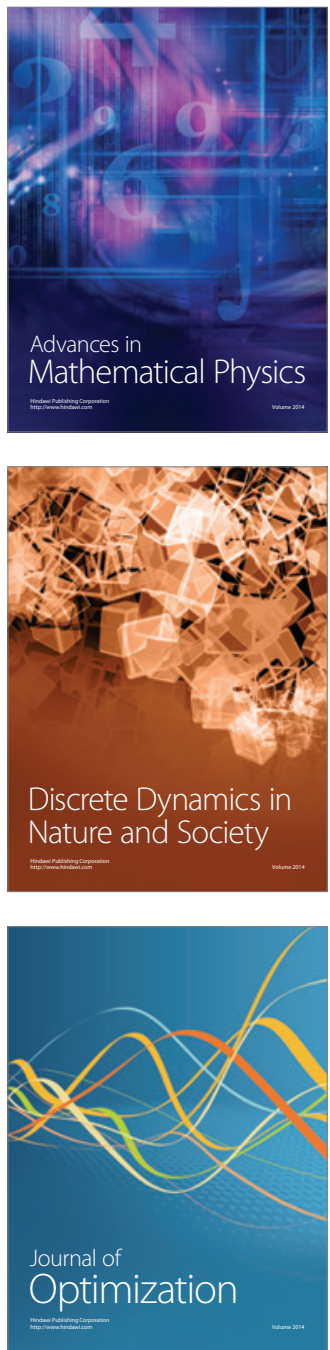